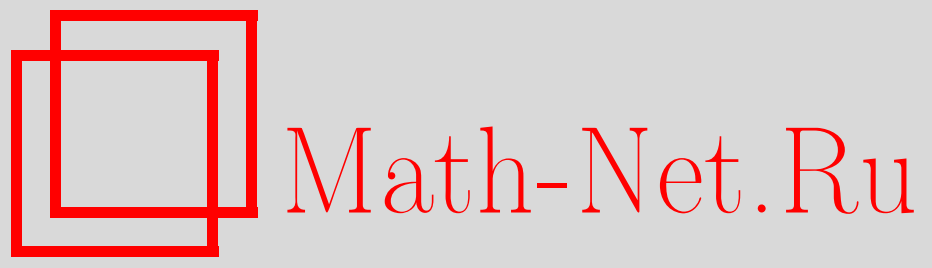

$\mathrm{X}$. Chen, Scaling limit of the path leading to the leftmost particle in a branching random walk, Теория вероятн. и ее примен., 2014, том 59, выпуск 4, 727-751

DOI: https://doi.org/10.4213/tvp4593

Использование Общероссийского математического портала Math-Net.Ru подразумевает, что вы прочитали и согласны с пользовательским соглашением http://www . mathnet.ru/rus/agreement

Параметры загрузки:

IP: 54.162 .127 .20

26 апреля 2023 г., 17:47:52

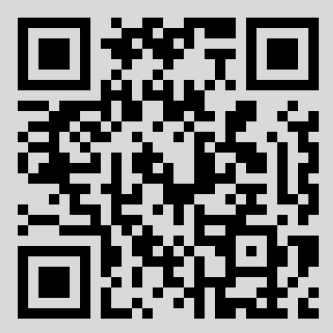




\title{
SCALING LIMIT OF THE PATH LEADING TO THE LEFTMOST PARTICLE IN A BRANCHING RANDOM WALK
}

\begin{abstract}
Рассматривается граничный случай надкритического ветвящегося случайного блуждания с дискретным временем на действительной прямой. Известно, что при условии невырождения этой системы положение самой левой частицы $n$-го поколения ведет себя асимптотически как $3 \ln n / 2$. Цель данной работы - доказать, что путь от корня к самой левой частице сходится, после соответствующей нормировки, к броуновской экскурсии в пространстве $D([0,1], \mathbf{R})$.
\end{abstract}

Ключевые слова и фразы: ветвящееся случайное блуждание, спинальное разложение.

1. Introduction. We consider a branching random walk, which is constructed according to a point process $\mathscr{L}$ on the line. Precisely speaking, the system is started with one initial particle at the origin. This particle is called the root, denoted by $\varnothing$. At time 1 , the root dies and gives birth to some new particles, which form the first generation. Their positions constitute a point process distributed as $\mathscr{L}$. At time 2, each of these particles dies and gives birth to new particles whose positions - relative to that of their parent - constitute a new independent copy of $\mathscr{L}$. The system grows according to the same mechanism.

We denote by $\mathbb{T}$ the genealogical tree of the system, which is clearly a Galton-Watson tree rooted at $\varnothing$. If a vertex $u \in \mathbb{T}$ is in the $n$-th generation, we write $|u|=n$ and denote its position by $V(u)$. Then $\{V(u),|u|=1\}$ follows the same law as $\mathscr{L}$. The family of positions $(V(u) ; u \in \mathbb{T})$ is viewed as our branching random walk.

Throughout the paper, the branching random walk is assumed to be in the boundary case (see [5]):

$$
\mathbf{E}\left[\sum_{|u|=1} 1\right]>1, \quad \mathbf{E}\left[\sum_{|x|=1} \mathrm{e}^{-V(x)}\right]=1, \quad \mathbf{E}\left[\sum_{|x|=1} V(x) \mathrm{e}^{-V(x)}\right]=0 .
$$

\footnotetext{
${ }^{*}$ Institut Camille Jordan, Université Claude Bernard Lyon 1, 43 Boulevard du 11 novembre 1918, 69622 Villeurbanne cedex, France; e-mail: xchen@math.univ_lyon1.fr
} 
For any $y \in \mathbf{R}$, let $y_{+}:=\max \{y, 0\}$ and $\log _{+} y:=\log (\max \{y, 1\})$. We also assume the following integrability conditions:

$$
\begin{gathered}
\mathbf{E}\left[\sum_{|u|=1} V(u)^{2} \mathrm{e}^{-V(u)}\right]<\infty, \\
\mathbf{E}\left[X\left(\log _{+} X\right)^{2}\right]<\infty, \quad \mathbf{E}\left[\widetilde{X} \log _{+} \tilde{X}\right]<\infty,
\end{gathered}
$$

where

$$
X:=\sum_{|u|=1} \mathrm{e}^{-V(u)}, \quad \tilde{X}:=\sum_{|u|=1} V(u)_{+} \mathrm{e}^{-V(u)} .
$$

We define $I_{n}$ to be the leftmost position in the $n$-th generation, i.e.,

$$
I_{n}:=\inf \{V(u),|u|=n\},
$$

with inf $0:=\infty$. If $I_{n}<\infty$, we choose a vertex uniformly in the set $\{u:|u|=$ $\left.n, V(u)=I_{n}\right\}$ of leftmost particles at time $n$ and denote it by $m^{(n)}$. We let $\llbracket \varnothing, m^{(n)} \rrbracket=\left\{\varnothing=: m_{0}^{(n)}, m_{1}^{(n)}, \ldots, m_{n}^{(n)}:=m^{(n)}\right\}$ be the shortest path in $\mathbb{T}$ relating the root $\varnothing$ to $m^{(n)}$, and introduce the path from the root to $m^{(n)}$ as follows

$$
\left(I_{n}(k) ; 0 \leqslant k \leqslant n\right):=\left(V\left(m_{k}^{(n)}\right) ; 0 \leqslant k \leqslant n\right) .
$$

In particular, $I_{n}(0)=0$ and $I_{n}(n)=I_{n}$. Let $\sigma$ be the positive real number such that $\sigma^{2}=\mathbf{E}\left[\sum_{|u|=1} V(u)^{2} \mathrm{e}^{-V(u)}\right]$. Our main result is as follows.

Theorem 1.1. The rescaled path $\left(I_{n}(\lfloor s n\rfloor) / \sigma \sqrt{n} ; 0 \leqslant s \leqslant 1\right)$ converges in law in $D([0,1], \mathbf{R})$, to a normalized Brownian excursion $\left(e_{s} ; 0 \leqslant s \leqslant 1\right)$.

$\mathrm{R}$ e $\mathrm{m}$ a r k 1.1. It has been proved in [1], [11], and [2] that $I_{n}$ is around $3 \ln n / 2$. In [3], the authors proved that, for the model of branching Brownian motion, the time reversed path followed by the leftmost particle converges in law to a certain stochastic process.

Let us say a few words about the proof of Theorem 1.1. We first consider the path leading to $m^{(n)}$, by conditioning that its ending point $I_{n}$ is located atypically below $3 \ln n / 2-z$ with large $z$. Then we apply the well-known spinal decomposition to show that this path, conditioned to $\left\{I_{n} \leqslant 3 \ln n / 2-z\right\}$, behaves like a simple random walk staying positive but tied down at the end. Such a random walk, being rescaled, converges in law to the Brownian excursion (see [9]). We then prove our main result by removing the condition of $I_{n}$. The main strategy is borrowed from [2], but with appropriate refinements.

The rest of the paper is organized as follows. In section 2, we recall the spinal decomposition by a change of measures, which implies the useful many-to-one lemma. We prove a conditioned version of Theorem $1.1 \mathrm{in}$ section 3 . In section 4 , we remove the conditioning and prove the theorem.

Throughout the paper, we use $a_{n} \sim b_{n}(n \rightarrow \infty)$ to denote $\lim _{n \rightarrow \infty}\left(a_{n} / b_{n}\right)=1$; and let $\left(c_{i}\right)_{i \geqslant 0}$ denote finite and positive constants. We write $\mathbf{E}[f ; A]$ for $\mathbf{E}\left[f \mathbf{1}_{A}\right]$. Moreover, $\sum_{\varnothing}:=0$ and $\prod_{\varnothing}:=1$. 


\section{Lyons' change of measures and spinal decomposition.}

For any $a \in \mathbf{R}$, let $\mathbf{P}_{a}$ be the probability measure such that $\mathbf{P}_{a}((V(u), u \in \mathbb{T}) \in \cdot)=\mathbf{P}((V(u)+a, u \in \mathbb{T}) \in \cdot)$. The corresponding expectation is denoted by $\mathbf{E}_{a}$. Let $\left(\mathscr{F}_{n}, n \geqslant 0\right)$ be the natural filtration generated by the branching random walk and let $\mathscr{F}_{\infty}:=\vee_{n \geqslant 0} \mathscr{F}_{n}$. We introduce the following random variables:

$$
W_{n}:=\sum_{|u|=n} e^{-V(u)}, \quad n \geqslant 0 .
$$

It follows immediately from (1.1) that $\left(W_{n}, n \geqslant 0\right)$ is a nonnegative martingale with respect to $\left(\mathscr{F}_{n}\right)$. It is usually referred as the additive martingale. We define a probability measure $\mathbf{Q}_{a}$ on $\mathscr{F}_{\infty}$ such that for any $n \geqslant 0$,

$$
\left.\frac{d \mathbf{Q}_{a}}{d \mathbf{P}_{a}}\right|_{\mathscr{F}_{n}}:=e^{a} W_{n} .
$$

For convenience, we write $\mathbf{Q}$ for $\mathbf{Q}_{0}$.

Let us give the description of the branching random walk under $\mathbf{Q}_{a}$ in an intuitive way, which is known as the spinal decomposition. We introduce another point process $\widehat{\mathscr{L}}$ with Radon-Nykodin derivative $\sum_{x \in \mathscr{L}} e^{-x}$ with respect to the law of $\mathscr{L}$. Under $\mathbf{Q}_{a}$, the branching random walk evolves as follows. Initially, there is one particle $w_{0}$ located at $V\left(w_{0}\right)=a$. At each step $n$, particles at generation $n$ die and give birth to new particles independently according to the law of $\mathscr{L}$, except for the particle $w_{n}$ which generates its children according to the law of $\widehat{\mathscr{L}}$. The particle $w_{n+1}$ is chosen proportionally to $e^{-V(u)}$ among the children $u$ of $w_{n}$. We still call $\mathbb{T}$ the genealogical tree of the process, so that $\left(w_{n}\right)_{n \geqslant 0}$ is a ray in $\mathbb{T}$, which is called the spine. This change of probabilities was presented in various forms; see, for example [15], [11], and [8].

It is convenient to use the following notation. For any $u \in \mathbb{T} \backslash\{\varnothing\}$, let $\overleftarrow{u}$ be the parent of $u$, and

$$
\Delta V(u):=V(u)-V(\overleftarrow{u})
$$

Let $\Omega(u)$ be the set of brothers of $u$, i.e., $\Omega(u):=\{v \in \mathbb{T}: \overleftarrow{v}=\overleftarrow{u}, v \neq u\}$ Let $\delta$ denote the Dirac measure. Then under $\mathbf{Q}_{a}, \sum_{|u|=1} \delta_{\Delta V(u)}$ follows the law of $\widehat{\mathscr{L}}$. Further, we recall the following proposition, from [11] and [15].

Proposition 2.1. (1) For any $|u|=n$, we have

$$
\mathbf{Q}_{a}\left[w_{n}=u \mid \mathscr{F}_{n}\right]=\frac{e^{-V(u)}}{W_{n}} .
$$

(2) Under $\mathbf{Q}_{a}$, the random variables $\left(\sum_{v \in \Omega\left(w_{n}\right)} \delta_{\Delta V(v)}, \Delta V\left(w_{n}\right)\right), n \geqslant 1$, are independent and identically distributed. 
As a consequence of this proposition, we get the many-to-one lemma as follows.

Lemma 2.1. There exists a centered random walk $\left(S_{n} ; n \geqslant 0\right)$ with $\mathbf{P}_{a}\left(S_{0}=a\right)=1$ such that for any $n \geqslant 1$ and any measurable function $g: \mathbf{R}^{n} \rightarrow[0, \infty)$, we have

$$
\mathbf{E}_{a}\left[\sum_{|u|=n} g\left(V\left(u_{1}\right), \ldots, V\left(u_{n}\right)\right)\right]=\mathbf{E}_{a}\left[e^{S_{n}-a} g\left(S_{1}, \ldots, S_{n}\right)\right],
$$

where we denote by $\llbracket \varnothing, u \rrbracket=\left\{\varnothing=: u_{0}, u_{1} \ldots, u_{|u|}:=u\right\}$ the ancestral line of $u$ in $\mathbb{T}$.

Note that by (1.3), $S_{1}$ has the finite variance $\sigma^{2}=\mathbf{E}\left[S_{1}^{2}\right]=$ $\mathbf{E}\left[\sum_{|u|=1} V(u)^{2} e^{-V(u)}\right]$.

2.1. Convergence in law for the one-dimensional random walk. Let us introduce some results about the centered random walk $\left(S_{n}\right)$ with finite variance, which will be used later. For any $0 \leqslant m \leqslant n$, we define $\underline{S}_{[m, n]}:=\min _{m \leqslant j \leqslant n} S_{j}$, and $\underline{S}_{n}=\underline{S}_{[0, n]}$. We denote by $R(x)$ the renewal function of $\left(S_{n}\right)$, which is defined as follows:

$$
R(x)=\mathbf{1}_{\{x=0\}}+\mathbf{1}_{\{x>0\}} \sum_{k \geqslant 0} \mathbf{P}\left(-x \leqslant S_{k}<\underline{S}_{n-1}\right) .
$$

For the random walk $\left(-S_{n}\right)$, we define $\underline{S}_{[m, n]}^{-}, \underline{S}_{n}^{-}$, and $R_{-}(x)$ similarly. It is known (see $\left[10\right.$, p. 360]) that there exists $c_{0}>0$ such that

$$
\lim _{x \rightarrow \infty} \frac{R(x)}{x}=c_{0} .
$$

Moreover, it is shown in [13] that there exist $C_{+}, C_{-}>0$ such that for any $a \geqslant 0$,

$$
\begin{aligned}
\mathbf{P}_{a}\left(\underline{S}_{n} \geqslant 0\right) & \sim \frac{C_{+}}{\sqrt{n}} R(a), \\
\mathbf{P}_{a}\left(\underline{S}_{n}^{-} \geqslant 0\right) & \sim \frac{C_{-}}{\sqrt{n}} R_{-}(a) .
\end{aligned}
$$

We also state the following inequalities (see Lemmas 2.2 and 2.4 in [4], respectively).

$\mathrm{F}$ a c t 2.1. (i) There exists a constant $c_{1}>0$ such that for any $b \geqslant$ $a \geqslant 0, x \geqslant 0$ and $n \geqslant 1$,

$$
\mathbf{P}\left(\underline{S}_{n} \geqslant-x ; S_{n} \in[a-x, b-x]\right) \leqslant c_{1}(1+x)(1+b-a)(1+b) n^{-3 / 2} .
$$

(ii) Let $0<\lambda<1$. There exists a constant $c_{2}>0$ such that for any $b \geqslant a \geqslant 0, x, y \geqslant 0$, and $n \geqslant 1$,

$\mathbf{P}_{x}\left(S_{n} \in[y+a, y+b], \underline{S}_{n} \geqslant 0, \underline{S}_{[\lambda n, n]} \geqslant y\right) \leqslant c_{2}(1+x)(1+b-a)(1+b) n^{-3 / 2}$. 
Before we give the following lemma, we recall the definition of lattice distribution (see [10, p. 138]). The distribution of a random variable $X_{1}$ is lattice, if it is concentrated on a set of points $\alpha+\beta \mathbf{Z}$, with $\alpha$ arbitrary. The largest $\beta$ satisfying this property is called the span of $X_{1}$. Otherwise, the distribution of $X_{1}$ is called nonlattice.

Lemma 2.2. Let $\left(r_{n}\right)_{n \geqslant 0}$ be a sequence of real numbers such that $\lim _{n \rightarrow \infty} r_{n} / \sqrt{n}=0$. Let $f: \mathbf{R}_{+} \rightarrow \mathbf{R}$ be a Riemann integrable function. We suppose that there exists a nonincreasing function $\bar{f}: \mathbf{R}_{+} \rightarrow \mathbf{R}$ such that $|f(x)| \leqslant \bar{f}(x)$ for any $x \geqslant 0$ and $\int_{x \geqslant 0} x \bar{f}(x) d x<\infty$. For $0<\Delta<1$, let $F: D([0, \Delta], \mathbf{R}) \rightarrow[0,1]$ be continuous. Let $a \geqslant 0$.

(I) Nonlattice case. If the distribution of $\left(S_{1}-S_{0}\right)$ is nonlattice, then there exists a constant $C_{1}>0$ such that

$$
\begin{aligned}
\lim _{n \rightarrow \infty} & n^{3 / 2} \mathbf{E}\left[F\left(\frac{S_{\lfloor s n\rfloor}}{\sigma \sqrt{n}} ; 0 \leqslant s \leqslant \Delta\right) f\left(S_{n}-y\right) ; \underline{S}_{n} \geqslant-a, \underline{S}_{[\Delta n, n]} \geqslant y\right] \\
= & C_{1} R(a) \int_{x \geqslant 0} f(x) R_{-}(x) d x \mathbf{E}\left[F\left(e_{s} ; 0 \leqslant s \leqslant \Delta\right)\right],
\end{aligned}
$$

uniformly in $y \in\left[0, r_{n}\right]$.

(II) Lattice case. If the distribution of $\left(S_{1}-S_{0}\right)$ is supported in $(\alpha+\beta \mathbf{Z})$ with span $\beta$, then for any $d \in \mathbf{R}$,

$$
\begin{gathered}
\lim _{n \rightarrow \infty} n^{3 / 2} \mathbf{E}\left[F\left(\frac{S_{\lfloor s n\rfloor}}{\sigma \sqrt{n}} ; 0 \leqslant s \leqslant \Delta\right) f\left(S_{n}-y+d\right) ; \underline{S}_{n} \geqslant-a, \underline{S}_{[\Delta n, n]} \geqslant y-d\right] \\
=C_{1} R(a) \beta \sum_{j \geqslant\lceil-d / \beta\rceil} f(\beta j+d) R_{-}(\beta j+d) \mathbf{E}\left[F\left(e_{s} ; 0 \leqslant s \leqslant \Delta\right)\right] .
\end{gathered}
$$

uniformly in $y \in\left[0, r_{n}\right] \cap\{\alpha n+\beta \mathbf{Z}\}$.

$\mathrm{P} \mathrm{r}$ o of of $\mathrm{L} \mathrm{e} \mathrm{m} \mathrm{m}$ a 2.2. The lemma is a refinement of Lemma 2.3 in [2], which proved the convergence in the nonlattice case when $a=0$ and $F \equiv 1$. We consider the nonlattice case first. We denote the expectation on the left-hand side of $(2.11)$ by $\chi(F, f)$. Observe that for any $K \in \mathbf{N}_{+}$,

$$
\chi(F, f)=\chi\left(F, f(x) 1_{(0 \leqslant x \leqslant K)}\right)+\chi\left(F, f(x) 1_{(x>K)}\right) .
$$

Since $0 \leqslant F \leqslant 1$, we have $\chi\left(F, f(x) 1_{(x>K)}\right) \leqslant \chi\left(1, f(x) 1_{(x>K)}\right)$, which is bounded by

$$
\begin{aligned}
\sum_{j \geqslant K} \mathbf{E}_{a}[ & f\left(S_{n}-y-a\right) ; \underline{S}_{n} \geqslant 0, \\
& \left.\underline{S}_{[\Delta n, n]} \geqslant y+a, S_{n} \in[y+a+j, y+a+j+1]\right] .
\end{aligned}
$$

Recall that $|f(x)| \leqslant \bar{f}(x)$ with $\bar{f}$ nonincreasing. We get that

$$
\begin{aligned}
\chi\left(1, f(x) 1_{(x>K)}\right) \leqslant \sum_{j \geqslant K} \bar{f}(j) \mathbf{P}_{a}\left[\underline{S}_{n} \geqslant 0, \underline{S}_{[\Delta n, n]} \geqslant y+a,\right. \\
\left.S_{n} \in[y+a+j, y+a+j+1]\right] .
\end{aligned}
$$


It then follows from (2.10) that

$$
\chi\left(1, f(x) 1_{(x>K)}\right) \leqslant 2 c_{2}(1+a)\left(\sum_{j \geqslant K} \bar{f}(j)(2+j)\right) n^{-3 / 2} .
$$

Since $\int_{0}^{\infty} x \bar{f}(x) d x<\infty$, the sum $\sum_{j \geqslant K} \bar{f}(j)(2+j)$ decreases to zero as $K \uparrow \infty$. We thus only need to estimate $\chi\left(F, f(x) 1_{(0 \leqslant x \leqslant K)}\right)$. Note that $f$ is Riemann integrable. It suffices to consider $\chi\left(F, 1_{(0 \leqslant x \leqslant K)}\right)$ with $K$ a positive constant.

Applying the Markov property at time $\lfloor\Delta n\rfloor$ shows that

$$
\begin{aligned}
\chi\left(F, 1_{(0 \leqslant x \leqslant K)}\right)= & \mathbf{E}_{a}\left[F\left(\frac{S_{\lfloor s n\rfloor}-a}{\sigma \sqrt{n}} ; 0 \leqslant s \leqslant \Delta\right) ;\right. \\
& \left.S_{n} \leqslant y+a+K, \underline{S}_{n} \geqslant 0, \underline{S}_{[\Delta n, n]} \geqslant y+a\right] \\
= & \mathbf{E}_{a}\left[F\left(\frac{S_{\lfloor s n\rfloor}-a}{\sigma \sqrt{n}} ; 0 \leqslant s \leqslant \Delta\right) \Psi_{K}\left(S_{\lfloor\Delta n\rfloor}\right) ; \underline{S}_{\lfloor\Delta n\rfloor} \geqslant 0\right],
\end{aligned}
$$

where $\Psi_{K}(x):=\mathbf{P}_{x}\left[S_{n-\lfloor\Delta n\rfloor} \leqslant y+a+K, \underline{S}_{n-\lfloor\Delta n\rfloor} \geqslant y+a\right]$. By reversing time, we obtain that $\Psi_{K}(x)=\mathbf{P}\left[\underline{S}_{m}^{-} \geqslant\left(-S_{m}\right)+(y+a-x) \geqslant-K\right]$ with $m:=n-\lfloor\Delta n\rfloor$.

We define $\tau_{n}$ as the first time when the random walk $(-S)$ hits the minimal level during $[0, n]$, namely, $\tau_{n}:=\inf \left\{k \in[0, n]:-S_{k}=\underline{S}_{n}^{-}\right\}$. Define also $\varkappa(z, \zeta ; n):=\mathbf{P}\left(-S_{n} \in[z, z+\zeta], \underline{S}_{n}^{-} \geqslant 0\right)$ for any $z, \zeta \geqslant 0$. Then,

$$
\begin{aligned}
\Psi_{K}(x) & =\sum_{k=0}^{m} \mathbf{P}\left[\tau_{m}=k ; \underline{S}_{m}^{-} \geqslant\left(-S_{m}\right)+(y+a-x) \geqslant-K\right] \\
& =\sum_{k=0}^{m} \mathbf{P}\left[-S_{k}=\underline{S}_{k}^{-} \geqslant-K ; \varkappa\left(x-y-a, \underline{S}_{k}^{-}+K ; m-k\right)\right],
\end{aligned}
$$

where the last equality follows from the Markov property.

Let $\psi(x):=x e^{-x^{2} / 2} \mathbf{1}_{(x \geqslant 0)}$. Combining Theorem 1 of [6] with (2.7) yields that

$$
\varkappa(z, \zeta ; n)=\mathbf{P}_{0}\left[-S_{n} \in[z, z+\zeta] ; \underline{S}_{n} \geqslant 0\right]=\frac{C_{-} \zeta}{\sigma n} \psi\left(\frac{z}{\sigma \sqrt{n}}\right)+o\left(n^{-1}\right),
$$

uniformly in $z \in \mathbf{R}_{+}$and $\zeta$ in compact sets of $\mathbf{R}_{+}$. Note that $\psi$ is bounded on $\mathbf{R}_{+}$. Therefore, there exists a constant $c_{3}>0$ such that for any $\zeta \in[0, K]$, $z \geqslant 0$ and $n \geqslant 0$

$$
\varkappa(z, \zeta ; n) \leqslant c_{3} \frac{(1+K)}{n+1} .
$$


Let $k_{n}:=\lfloor\sqrt{n}\rfloor$. We divide the sum on the right-hand side of $(2.15)$ into two parts:

$$
\Psi_{K}(x)=\sum_{k=0}^{k_{n}}+\sum_{k=k_{n}+1}^{m} \mathbf{P}\left[-S_{k}=\underline{S}_{k}^{-} \geqslant-K ; \varkappa\left(x-y-a, \underline{S}_{k}^{-}+K ; m-k\right)\right] .
$$

By (2.16), under the assumption that $y=o(\sqrt{n})$, the first part becomes

$$
\begin{gathered}
\frac{C_{-}}{\sigma m} \psi\left(\frac{x-a}{\sigma \sqrt{m}}\right) \sum_{k=0}^{k_{n}} \mathbf{E}\left[\underline{S}_{k}^{-}+K ;-S_{k}=\underline{S}_{k}^{-} \geqslant-K\right] \\
+o\left(n^{-1}\right) \sum_{k=0}^{k_{n}} \mathbf{P}\left[-S_{k}=\underline{S}_{k}^{-} \geqslant-K\right] \\
=\frac{C_{-}}{\sigma m} \psi\left(\frac{x-a}{\sigma \sqrt{m}}\right) \int_{0}^{K} R_{-}(u) d u+o\left(n^{-1}\right),
\end{gathered}
$$

where the last equation comes from the fact that $\sum_{k \geqslant 0} \mathbf{E}\left[\underline{S}_{k}^{-}+K ;-S_{k}=\right.$ $\left.\underline{S}_{k}^{-} \geqslant-K\right]=\int_{0}^{K} R_{-}(u) d u$. On the other hand, using (2.17) for $\varkappa(x-y-$ $\left.a, \underline{S}_{k}^{-}+K ; m-k\right)$ and then applying (i) of Fact 2.1 imply that for $n$ large enough, the second part of (2.18) is bounded by

$$
\begin{aligned}
\sum_{k=k_{n}+1}^{m} c_{3} & \frac{1+K}{m+1-k} \mathbf{P}\left(\underline{S}_{k}^{-} \geqslant-K,-S_{k} \in[-K, 0]\right) \\
\leqslant c_{4} \sum_{k=k_{n}+1}^{m} \frac{(1+K)^{3}}{(m+1-k) k^{3 / 2}} & =o\left(n^{-1}\right) .
\end{aligned}
$$

By (2.19) and (2.20), we obtain that as $n$ goes to infinity,

$$
\Psi_{K}(x)=o\left(n^{-1}\right)+\frac{C_{-}}{\sigma(n-\lfloor\Delta n\rfloor)} \psi\left(\frac{x-a}{\sigma \sqrt{n-\lfloor\Delta n\rfloor}}\right) \int_{0}^{K} R_{-}(u) d u
$$

uniformly in $x \geqslant 0$ and $y \in\left[0, r_{n}\right]$. Plugging it into (2.14) and then combining with (2.7) yield that

$$
\begin{aligned}
\chi\left(F, 1_{(0 \leqslant x \leqslant K)}\right)=o\left(n^{-3 / 2}\right)+\frac{C_{-}}{\sigma(1-\Delta) n} \int_{0}^{K} R_{-}(u) d u \\
\times \frac{C_{+} R(a)}{\sqrt{\Delta n}} \mathbf{E}_{a}\left[F\left(\frac{S_{\lfloor s n\rfloor}-a}{\sigma \sqrt{n}} ; 0 \leqslant s \leqslant \Delta\right)\right. \\
\left.\quad \times \psi\left(\frac{S_{\Delta n}-a}{\sigma \sqrt{(1-\Delta) n}}\right) \mid \underline{S}_{\Delta n} \geqslant 0\right] .
\end{aligned}
$$

Theorem 1.1 of [7] says that under the conditioned probability

$$
\mathbf{P}_{a}\left(\cdot \mid \underline{S}_{\Delta n} \geqslant 0\right), \quad\left(\frac{S_{\lfloor r \Delta n\rfloor}}{\sigma \sqrt{\Delta n}} ; 0 \leqslant r \leqslant 1\right)
$$


converges in law to a Brownian meander, denoted by $\left(\mathscr{M}_{r} ; 0 \leqslant r \leqslant 1\right)$. Therefore,

$$
\begin{aligned}
\chi\left(F, 1_{(0 \leqslant x \leqslant K)}\right) \sim & \frac{C_{-} C_{+} R(a)}{\sigma n^{3 / 2}(1-\Delta) \sqrt{\Delta}} \int_{0}^{K} R_{-}(u) d u \\
& \times \mathbf{E}\left[F\left(\sqrt{\Delta} \mathscr{M}_{s / \Delta} ; 0 \leqslant s \leqslant \Delta\right) \psi\left(\frac{\sqrt{\Delta} \mathscr{M}_{1}}{\sqrt{1-\Delta}}\right)\right] .
\end{aligned}
$$

It remains to check that

$$
\begin{aligned}
\frac{1}{(1-\Delta) \sqrt{\Delta}} & \mathbf{E}\left[F\left(\sqrt{\Delta} \mathscr{M}_{s / \Delta} ; 0 \leqslant s \leqslant \Delta\right) \psi\left(\frac{\sqrt{\Delta} \mathscr{M}_{1}}{\sqrt{1-\Delta}}\right)\right] \\
& =\sqrt{\frac{\pi}{2}} \mathbf{E}\left[F\left(e_{s} ; 0 \leqslant s \leqslant \Delta\right)\right] .
\end{aligned}
$$

Let $\left(R_{s} ; 0 \leqslant s \leqslant 1\right)$ be a standard three-dimensional Bessel process. Then, as is shown in [12],

$$
\begin{aligned}
& \frac{1}{(1-\Delta) \sqrt{\Delta}} \mathbf{E}\left[F\left(\sqrt{\Delta} \mathscr{M}_{s / \Delta} ; 0 \leqslant s \leqslant \Delta\right) \psi\left(\frac{\sqrt{\Delta} \mathscr{M}_{1}}{\sqrt{1-\Delta}}\right)\right] \\
& =\sqrt{\frac{\pi}{2}} \frac{1}{(1-\Delta) \sqrt{\Delta}} \mathbf{E}\left[\frac{1}{R_{1}} F\left(\sqrt{\Delta} R_{s / \Delta} ; 0 \leqslant s \leqslant \Delta\right) \psi\left(\frac{\sqrt{\Delta} R_{1}}{\sqrt{1-\Delta}}\right)\right] \\
& =\sqrt{\frac{\pi}{2}} \mathbf{E}\left[\frac{1}{(1-\Delta)^{3 / 2}} e^{-R_{\Delta}^{2} / 2(1-\Delta)} F\left(R_{s} ; 0 \leqslant s \leqslant \Delta\right)\right],
\end{aligned}
$$

where the last equation follows from the scaling property of Bessel process. Let $\left(r_{s} ; 0 \leqslant s \leqslant 1\right)$ be a standard three-dimensional Bessel bridge. Note that for any $\Delta<1,\left(r_{s} ; 0 \leqslant s \leqslant \Delta\right)$ is equivalent to $\left(R_{s} ; 0 \leqslant s \leqslant \Delta\right)$, with density $(1-\Delta)^{-3 / 2} \exp \left(-R_{\Delta}^{2} /(2(1-\Delta))\right)$ (see [16, p. 468, equation (3.11)]). Thus,

$$
\begin{aligned}
\frac{1}{(1-\Delta) \sqrt{\Delta}} & \mathbf{E}\left[F\left(\sqrt{\Delta} \mathscr{M}_{s / \Delta} ; 0 \leqslant s \leqslant \Delta\right) \psi\left(\frac{\sqrt{\Delta} \mathscr{M}_{1}}{\sqrt{1-\Delta}}\right)\right] \\
& =\sqrt{\frac{\pi}{2}} \mathbf{E}\left[F\left(r_{s} ; 0 \leqslant s \leqslant \Delta\right)\right] .
\end{aligned}
$$

Since a normalized Brownian excursion is exactly a standard threedimensional Bessel bridge, this yields (2.23). Therefore, (2.11) is proved by taking $C_{1}=\sqrt{\pi / 2}\left(C_{-} C_{+} / \sigma\right)$.

The proof of the lemma in the lattice case is along the same lines, except that we use Theorem 2 (instead of Theorem 1) of [6]. 
3. Conditioning on the event $\left\{I_{n} \leqslant 3 \ln n / 2-z\right\}$. On the event $\left\{I_{n} \leqslant 3 \ln n / 2-z\right\}$, we analyze the sample path leading to a particle located at the leftmost position at the $n$th generation. For $z \geqslant 0$ and $n \geqslant 1$, let $a_{n}(z):=3 \ln n / 2-z$ if the distribution of $\mathscr{L}$ is nonlattice and let $a_{n}(z):=$ $\alpha n+\beta\lfloor(3 \ln n / 2-\alpha n) / \beta\rfloor-z$ if the distribution of $\mathscr{L}$ is supported by $\alpha+\beta \mathbf{Z}$. This section is devoted to the proof of the following proposition.

Proposition 3.1. For any $\Delta \in(0,1]$ and any continuous functional $F: D([0, \Delta], \mathbf{R}) \rightarrow[0,1]$,

$$
\begin{aligned}
\lim _{z \rightarrow \infty} \limsup _{n \rightarrow \infty} \mid \mathbf{E}\left[F\left(\frac{I_{n}(\lfloor s n\rfloor)}{\sigma \sqrt{n}} ; 0 \leqslant s \leqslant \Delta\right) \mid I_{n} \leqslant a_{n}(z)\right] \\
-\mathbf{E}\left[F\left(e_{s} ; 0 \leqslant s \leqslant \Delta\right)\right] \mid=0 .
\end{aligned}
$$

We begin with some preliminary results.

For any $0<\Delta<1$ and $L, K \geqslant 0$, we denote by $J_{z, K, L}^{\Delta}(n)$ the following collection of particles:

$$
\begin{gathered}
\left\{u \in \mathbb{T}:|u|=n, V(u) \leqslant a_{n}(z), \min _{0 \leqslant k \leqslant n} V\left(u_{k}\right) \geqslant-z+K,\right. \\
\left.\min _{\Delta n \leqslant k \leqslant n} V\left(u_{k}\right) \geqslant a_{n}(z+L)\right\} .
\end{gathered}
$$

Lemma 3.1. For any $\varepsilon>0$, there exists $L_{\varepsilon}>0$ such that for any $L \geqslant L_{\varepsilon}, n \geqslant 1$, and $z \geqslant K \geqslant 0$,

$$
\mathbf{P}\left(m^{(n)} \notin J_{z, K, L}^{\Delta}(n), I_{n} \leqslant a_{n}(z)\right) \leqslant\left(e^{K}+\varepsilon(1+z-K)\right) e^{-z} .
$$

P r o o f. It suffices to show that for any $\varepsilon \in(0,1)$, there exists $L_{\varepsilon} \geqslant 1$ such that for any $L \geqslant L_{\varepsilon}, n \geqslant 1$, and $z \geqslant K \geqslant 0$,

$$
\mathbf{P}\left(\exists|u|=n: V(u) \leqslant a_{n}(z), u \notin J_{z, K, L}^{\Delta}(n)\right) \leqslant\left(e^{K}+\varepsilon(1+z-K)\right) e^{-z} .
$$

We observe that

$$
\begin{gathered}
\mathbf{P}\left(\exists|u|=n: V(u) \leqslant a_{n}(z), u \notin J_{z, K, L}^{\Delta}(n)\right) \leqslant \mathbf{P}(\exists u \in \mathbb{T}: V(u) \leqslant-z+K) \\
+\mathbf{P}\left(\exists|u|=n: V(u) \leqslant a_{n}(z), \min _{0 \leqslant k \leqslant n} V\left(u_{k}\right) \geqslant-z+K\right. \\
\left.\min _{\Delta n \leqslant k \leqslant n} V\left(u_{k}\right) \leqslant a_{n}(z+L)\right) .
\end{gathered}
$$

On the one hand, by (2.4),

$$
\begin{aligned}
\mathbf{P}(\exists u \in \mathbb{T}: V(u) \leqslant-z+k) & \leqslant \sum_{n \geqslant 0} \mathbf{E}\left[\sum_{|u|=n} \mathbf{1}_{\left\{V(u) \leqslant-z+K<\min _{k<n} V\left(u_{k}\right)\right\}}\right] \\
& =\sum_{n \geqslant 0} \mathbf{E}\left[e^{S_{n}} ; S_{n} \leqslant-z+K<\underline{S}_{n-1}\right] \\
& \leqslant e^{-z+K} .
\end{aligned}
$$


On the other hand, denoting $A_{n}(z):=\left[a_{n}(z)-1, a_{n}(z)\right]$ for any $z \geqslant 0$,

$$
\begin{aligned}
& \mathbf{P}\left(\exists|u|=n: V(u) \leqslant a_{n}(z), \min _{0 \leqslant k \leqslant n} V\left(u_{k}\right) \geqslant-z+K,\right. \\
& \left.\min _{\Delta n \leqslant k \leqslant n} V\left(u_{k}\right) \leqslant a_{n}(z+L)\right) \\
& =\mathbf{P}_{z-K}\left(\exists|u|=n: V(u) \leqslant a_{n}(K), \min _{0 \leqslant k \leqslant n} V\left(u_{k}\right) \geqslant 0,\right. \\
& \left.\min _{\substack{\Delta \leqslant k \leqslant n\\
}} V\left(u_{k}\right) \leqslant a_{n}(K+L)\right) \\
& \sum_{\ell \geqslant L+K} \sum_{j=K}^{j=K+\ell} \mathbf{P}_{z-K}\left(\exists|u|=n: V(u) \in A_{n}(j), \min _{0 \leqslant k \leqslant n} V\left(u_{k}\right) \geqslant 0,\right. \\
& \left.\min _{\Delta n \leqslant k \leqslant n} V\left(u_{k}\right) \in A_{n}(\ell)\right) .
\end{aligned}
$$

According to Lemma 3.3 in [2], there exist constants $1>c_{5}>0$ and $c_{6}>0$ such that for any $n \geqslant 1, L \geqslant 0$, and $x, z \geqslant 0$,

$$
\begin{gathered}
\mathbf{P}_{x}\left(\exists u \in \mathbb{T}:|u|=n, V(u) \in A_{n}(z), \min _{0 \leqslant k \leqslant n} V\left(u_{k}\right) \geqslant 0,\right. \\
\left.\min _{\Delta n \leqslant k \leqslant n} V\left(u_{k}\right) \in A_{n}(z+L)\right) \\
\leqslant c_{6}(1+x) e^{-c_{5} L} e^{-x-z} .
\end{gathered}
$$

Hence, combining (3.6) with (3.5) yields that

$$
\begin{aligned}
\mathbf{P}(\exists|u|= & \left.n: V(u) \leqslant a_{n}(z), u \notin J_{z, K, L}^{\Delta}(n)\right) \\
& \leqslant e^{-z+K}+\sum_{\ell \geqslant L} \sum_{0 \leqslant j \leqslant \ell} c_{6}(1+z-K) e^{-c_{5}(\ell-j)} e^{-z-j} \\
& \leqslant\left(e^{K}+c_{7} \sum_{\ell \geqslant L} e^{-c_{5} \ell}(1+z-K)\right) e^{-z}
\end{aligned}
$$

where the last inequality comes from the fact that $\sum_{j \geqslant 0} e^{-\left(1-c_{5}\right) j}<\infty$. We take $L_{\varepsilon}=-c_{8} \ln \varepsilon$ so that $c_{7} \sum_{\ell \geqslant L} e^{-c_{5} \ell} \leqslant \varepsilon$ for all $L \geqslant L_{\varepsilon}$. Therefore, for any $L \geqslant L_{\varepsilon}, n \geqslant 1$, and $z \geqslant K \geqslant 0$,

$$
\mathbf{P}\left(\exists|u|=n: V(u) \leqslant a_{n}(z), u \notin J_{z, K, L}^{\Delta}(n)\right) \leqslant\left(e^{K}+\varepsilon(1+z-K)\right) e^{-z},
$$

which completes the proof.

For $b \in \mathbf{Z}_{+}$, we define

$$
\mathscr{E}_{n}=\mathscr{E}_{n}(z, b):=\left\{\forall k \leqslant n-b, \min _{u \geqslant w_{k},|u|=n} V(u)>a_{n}(z)\right\} .
$$

We note that on the event $\mathscr{E}_{n} \cap\left\{I_{n} \leqslant a_{n}(z)\right\}$, any particle located at the leftmost position must be separated from the spine after time $n-b$. 
Lemma 3.2. For any $\eta>0, L>0$, there exist $K(\eta)>0, B(L, \eta) \geqslant 1$, and $N(\eta) \geqslant 1$ such that for any $b \geqslant B(L, \eta), n \geqslant N(\eta)$, and $z \geqslant K \geqslant K(\eta)$,

$$
\mathbf{Q}\left(\mathscr{E}_{n}^{c}, w_{n} \in J_{z, K, L}^{\Delta}(n)\right) \leqslant \eta(1+L)^{2}(1+z-K) n^{-3 / 2} .
$$

We feel free to omit the proof of Lemma 3.2 since it is just a slightly stronger version of Lemma 3.8 in [2]. It follows from the same arguments.

Let us turn to the proof of Proposition 3.1. We break it up into 3 steps.

$\mathrm{S} \mathrm{t}$ e p I (The conditioned convergence of $\left(I_{n}(\lfloor s n\rfloor) / \sigma \sqrt{n} ; 0 \leqslant s \leqslant \Delta\right.$ ) for $\Delta<1$ in the nonlattice case).

Assume that the distribution of $\mathscr{L}$ is nonlattice in this step. Recall that $a_{n}(z)=3 \ln n / 2-z$. The tail distribution of $I_{n}$ has been given in Propositions 1.3 and 4.1 of [2], recalled as follows.

F a c t 3.1 (see [2]). There exists a constant $C>0$ such that

$$
\limsup _{z \rightarrow \infty} \limsup _{n \rightarrow \infty}\left|\frac{e^{z}}{z} \mathbf{P}\left(I_{n} \leqslant a_{n}(z)\right)-C\right|=0 .
$$

Furthermore, for any $\varepsilon>0$, there exist $N_{\varepsilon} \geqslant 1$ and $\Lambda_{\varepsilon}>0$ such that for any $n \geqslant N_{\varepsilon}$ and $\Lambda_{\varepsilon} \leqslant z \leqslant 3 \ln n / 2-\Lambda_{\varepsilon}$,

$$
\left|\frac{e^{z}}{z} \mathbf{P}\left(I_{n} \leqslant a_{n}(z)\right)-C\right| \leqslant \varepsilon
$$

For any continuous functional $F: D([0, \Delta], \mathbf{R}) \rightarrow[0,1]$, it is convenient to write that

$$
\Sigma_{n}(F, z):=\mathbf{E}\left[F\left(\frac{I_{n}(\lfloor s n\rfloor)}{\sigma \sqrt{n}} ; 0 \leqslant s \leqslant \Delta\right) \mathbf{1}_{\left\{I_{n} \leqslant a_{n}(z)\right\}}\right] .
$$

In particular, if $F \equiv 1$, then $\Sigma_{n}(1, z)=\mathbf{P}\left(I_{n} \leqslant a_{n}(z)\right)$. Thus,

$$
\frac{\Sigma_{n}(F, z)}{\Sigma_{n}(1, z)}=\mathbf{E}\left[F\left(\frac{I_{n}(\lfloor s n\rfloor)}{\sigma \sqrt{n}} ; 0 \leqslant s \leqslant \Delta\right) \mid I_{n} \leqslant a_{n}(z)\right] .
$$

Let us prove the following convergence for $0<\Delta<1$ :

$$
\operatorname{lims}_{z \rightarrow \infty} \limsup _{n \rightarrow \infty}\left|\frac{\Sigma_{n}(F, z)}{\Sigma_{n}(1, z)}-\mathbf{E}\left[F\left(e_{s}, 0 \leqslant s \leqslant \Delta\right)\right]\right|=0 .
$$

P r o of of (3.15). For any $n \geqslant 1, L \geqslant 0$ and $z \geqslant K \geqslant 0$, let

$$
\Pi_{n}(F)=\Pi_{n}(F, z, K, L):=\mathbf{E}\left[F\left(\frac{I_{n}(s n)}{\sigma \sqrt{n}} ; 0 \leqslant s \leqslant \Delta\right) \mathbf{1}_{\left\{m^{(n)} \in J_{z, K, L}^{\Delta}(n)\right\}}\right] .
$$

By Lemma 3.1, we obtain that for $L \geqslant L_{\varepsilon}, n \geqslant 1$, and $z \geqslant K \geqslant 0$,

$$
\left|\Sigma_{n}(F, z)-\Pi_{n}(F)\right| \leqslant\left(e^{K}+\varepsilon(1+z-K)\right) e^{-z} .
$$


Note that $m^{(n)}$ is chosen uniformly among the particles located at the leftmost position. Thus,

$$
\begin{aligned}
\Pi_{n}(F)= & \mathbf{E}\left[\sum_{|u|=n} \mathbf{1}_{\left(u=m^{(n)}, u \in J_{z, K, L}^{\Delta}(n)\right)} F\left(\frac{V\left(u_{\lfloor s n\rfloor}\right)}{\sigma \sqrt{n}} ; 0 \leqslant s \leqslant \Delta\right)\right] \\
=\mathbf{E}[ & \frac{1}{\sum_{|u|=n} \mathbf{1}_{\left(V(u)=I_{n}\right)}} \\
& \left.\quad \times \sum_{|u|=n} \mathbf{1}_{\left(V(u)=I_{n}, u \in J_{z, K, L}^{\Delta}(n)\right)} F\left(\frac{V\left(u_{\lfloor s n\rfloor}\right)}{\sigma \sqrt{n}} ; 0 \leqslant s \leqslant \Delta\right)\right] .
\end{aligned}
$$

Applying the change of measures given in (2.2), it follows from Proposition 2.1 that

$$
\begin{aligned}
\Pi_{n}(F)=\mathbf{E}_{\mathbf{Q}}[ & \frac{e^{V\left(w_{n}\right)}}{\sum_{|u|=n} \mathbf{1}_{\left(V(u)=I_{n}\right)}} \\
& \left.\times \mathbf{1}_{\left(V\left(w_{n}\right)=I_{n}, w_{n} \in J_{z, K, L}^{\Delta}(n)\right)} F\left(\frac{V\left(w_{\lfloor s n\rfloor}\right)}{\sigma \sqrt{n}} ; 0 \leqslant s \leqslant \Delta\right)\right] .
\end{aligned}
$$

In order to estimate $\Pi_{n}$, we restrict ourselves to the event $\mathscr{E}_{n}$. Define

$$
\begin{aligned}
\Lambda_{n}(F):=\mathbf{E}_{\mathbf{Q}}[ & \frac{e^{V\left(w_{n}\right)}}{\sum_{|u|=n} \mathbf{1}_{\left(V(u)=I_{n}\right)}} \\
& \left.\times \mathbf{1}_{\left(V\left(w_{n}\right)=I_{n}, w_{n} \in J_{z, K, L}^{\Delta}(n)\right)} F\left(\frac{V\left(w_{\lfloor s n\rfloor}\right)}{\sigma \sqrt{n}} ; 0 \leqslant s \leqslant \Delta\right) ; \mathscr{E}_{n}\right] .
\end{aligned}
$$

In view of Lemma 3.2 , for any $b \geqslant B(L, \eta), n \geqslant N(\eta)$, and $z \geqslant K \geqslant K(\eta)$,

$$
\begin{aligned}
\left|\Pi_{n}(F)-\Lambda_{n}(F)\right| & \leqslant \mathbf{E}_{\mathbf{Q}}\left[e^{V\left(w_{n}\right)} ; w_{n} \in J_{z, K, L}^{\Delta}(n), \mathscr{E}_{n}\right] \\
& \leqslant e^{-z} n^{-3 / 2} \mathbf{Q}\left(\mathscr{E}_{n}^{c}, w_{n} \in J_{z, K, L}^{\Delta}(n)\right) \\
& \leqslant \eta(1+L)^{2}(1+z-K) e^{-z} .
\end{aligned}
$$

On the event $\mathscr{E}_{n} \cap\left\{I_{n} \leqslant a_{n}(z)\right\}, \Lambda_{n}(F)$ equals

$$
\begin{gathered}
\mathbf{E}_{\mathbf{Q}}\left[\frac{e^{V\left(w_{n}\right)}}{\sum_{u>w_{n-b},|u|=n} \mathbf{1}_{\left(V(u)=I_{n}\right)}} \mathbf{1}_{\left(V\left(w_{n}\right)=I_{n}, w_{n} \in J_{z, K, L}^{\Delta}(n)\right)}\right. \\
\left.F\left(\frac{V\left(w_{\lfloor s n\rfloor}\right)}{\sigma \sqrt{n}} ; 0 \leqslant s \leqslant \Delta\right) ; \mathscr{E}_{n}\right] .
\end{gathered}
$$

Let, for $x \geqslant 0, L>0$, and $b \geqslant 1$,

$$
\begin{aligned}
f_{L, b}(x) & :=\mathbf{E}_{\mathbf{Q}_{x}}\left[\frac{e^{V\left(w_{b}\right)-L} \mathbf{1}_{\left\{V\left(w_{b}\right)=I_{b}\right\}}}{\sum_{|u|=b} \mathbf{1}_{\left\{V(u)=I_{b}\right\}}}, \min _{0 \leqslant k \leqslant b} V\left(w_{k}\right) \geqslant 0, V\left(w_{b}\right) \leqslant L\right] \\
& \leqslant \mathbf{Q}_{x}\left(\min _{0 \leqslant k \leqslant b} V\left(w_{k}\right) \geqslant 0, V\left(w_{b}\right) \leqslant L\right) .
\end{aligned}
$$


We choose $n$ large enough so that $\Delta n \leqslant n-b$. Thus, applying the Markov property at time $n-b$ yields that

$$
\begin{gathered}
\Lambda_{n}(F)=n^{3 / 2} e^{-z} \mathbf{E}_{\mathbf{Q}}\left[F\left(\frac{V\left(w_{\lfloor s n\rfloor}\right)}{\sigma \sqrt{n}} ; 0 \leqslant s \leqslant \Delta\right) f_{L, b}\left(V\left(w_{n-b}\right)-a_{n}(z+L)\right) ;\right. \\
\min _{0 \leqslant k \leqslant n-b} V\left(w_{k}\right) \geqslant-z+K, \\
\left.\min _{\Delta n \leqslant k \leqslant n-b} V\left(w_{k}\right) \geqslant a_{n}(z+L), \mathscr{E}_{n}\right] .
\end{gathered}
$$

Let us introduce the following quantity by removing the restriction to $\mathscr{E}_{n}$ :

$$
\begin{gathered}
\Lambda_{n}^{I}(F):=n^{3 / 2} e^{-z} \mathbf{E}_{\mathbf{Q}}\left[F\left(\frac{V\left(w_{\lfloor s n\rfloor}\right)}{\sigma \sqrt{n}} ; 0 \leqslant s \leqslant \Delta\right) f_{L, b}\left(V\left(w_{n-b}\right)-a_{n}(z+L)\right) ;\right. \\
\min _{0 \leqslant k \leqslant n-b} V\left(w_{k}\right) \geqslant-z+K, \\
\left.\min _{\Delta n \leqslant k \leqslant n-b} V\left(w_{k}\right) \geqslant a_{n}(z+L)\right] .
\end{gathered}
$$

We immediately observe that

$$
\begin{aligned}
\left|\Lambda_{n}(F)-\Lambda_{n}^{I}(F)\right| \leqslant n^{3 / 2} e^{-z} \mathbf{Q}\left(f_{L, b}\left(V\left(w_{n-b}\right)-a_{n}(z+L)\right),\right. & \\
\min _{0 \leqslant k \leqslant n-b} V\left(w_{k}\right) & \geqslant-z+K, \\
\min _{\Delta n \leqslant k \leqslant n-b} V\left(w_{k}\right) & \left.\geqslant a_{n}(z+L) ;\left(\mathscr{O}_{n}\right)^{c}\right) .
\end{aligned}
$$

By (3.20), we check that $\left|\Lambda_{n}(F)-\Lambda_{n}^{I}(F)\right| \leqslant n^{3 / 2} e^{-z} \mathbf{Q}\left(w_{n} \in J_{z, K, L}^{\Delta}(n),\left(\mathscr{E}_{n}\right)^{c}\right)$. Applying Lemma 3.2 again implies that

$$
\left|\Lambda_{n}(F)-\Lambda_{n}^{I}(F)\right| \leqslant \eta(1+L)^{2}(1+z-K) e^{-z} .
$$

Combining with (3.19), we obtain that for any $b \geqslant B(L, \eta), z \geqslant K \geqslant K(\eta)$, and $n$ large enough,

$$
\left|\Pi_{n}(F)-\Lambda_{n}^{I}(F)\right| \leqslant 2 \eta(1+L)^{2}(1+z-K) e^{-z} .
$$

Note that $\left(V\left(w_{k}\right) ; k \geqslant 1\right)$ is a centered random walk under $\mathbf{Q}$ and that it is proved in [2] that $f_{L, b}$ satisfies the conditions of Lemma 2.2. By (I) of Lemma 2.2, we get that

$$
\lim _{n \rightarrow \infty} \Lambda_{n}^{I}(F)=\alpha_{L, b}^{I} R(z-K) e^{-z} \mathbf{E}\left[F\left(e_{s}, 0 \leqslant s \leqslant \delta\right)\right],
$$

where $\alpha_{L, b}^{I}:=C_{1} \int_{x \geqslant 0} f_{L, b}(x) R_{-}(x) d x \in[0, \infty)$. Thus, by (3.25), one sees that for any $b \geqslant B(L, \eta)$ and $z \geqslant K \geqslant K(\eta)$,

$$
\begin{gathered}
\limsup _{n \rightarrow \infty}\left|\Pi_{n}(F)-\alpha_{L, b}^{I} R(z-K) e^{-z} \mathbf{E}\left[F\left(e_{s}, 0 \leqslant s \leqslant \Delta\right)\right]\right| \\
\leqslant 2 \eta(1+L)^{2}(1+z-K) e^{-z}
\end{gathered}
$$


Going back to (3.17), we deduce that for any $L \geqslant L_{\varepsilon}, b \geqslant B(L, \eta)$, and $z \geqslant K \geqslant K(\eta)$

$$
\begin{aligned}
& \limsup _{n \rightarrow \infty}\left|\Sigma_{n}(F, z)-\alpha_{L, b}^{I} R(z-K) e^{-z} \mathbf{E}\left[F\left(e_{s}, 0 \leqslant s \leqslant \Delta\right)\right]\right| \\
& \leqslant 2 \eta(1+L)^{2}(1+z-K) e^{-z}+\left(e^{K}+\varepsilon(1+z-K)\right) e^{-z} .
\end{aligned}
$$

Recall that $\lim _{z \rightarrow \infty} R(z) / z=c_{0}$. We multiply each term by $e^{z} / z$, and then let $z$ go to infinity to conclude that

$$
\limsup _{z \rightarrow \infty} \limsup _{n \rightarrow \infty}\left|\frac{e^{z}}{z} \Sigma_{n}(F, z)-\alpha_{L, b}^{I} c_{0} \mathbf{E}\left[F\left(e_{s}, 0 \leqslant s \leqslant \Delta\right)\right]\right| \leqslant 2 \eta(1+L)^{2}+\varepsilon .
$$

In particular, taking $F \equiv 1$ gives that

$$
\limsup _{z \rightarrow \infty} \limsup _{n \rightarrow \infty}\left|\frac{e^{z}}{z} \mathbf{P}\left(I_{n} \leqslant a_{n}(z)\right)-\alpha_{L, b}^{I} c_{0}\right| \leqslant 2 \eta(1+L)^{2}+\varepsilon .
$$

It follows from Fact 3.1 that $\left|C-\alpha_{L, b}^{I} c_{0}\right| \leqslant 2 \eta(1+L)^{2}+\varepsilon$. We thus choose $0<\varepsilon<C / 10$ and $0<\eta \leqslant \varepsilon /\left(2\left(1+L_{\varepsilon}\right)^{2}\right)$ so that $2 C>\alpha_{L_{\varepsilon}, b}^{I} c_{0}>C / 2>0$.

Therefore, for any $\varepsilon \in(0, C / 10), 0<\eta \leqslant \varepsilon /\left(2\left(1+L_{\varepsilon}\right)^{2}\right), L=L_{\varepsilon}$, and $b \geqslant B\left(L_{\varepsilon}, \eta\right)$,

$$
\limsup _{z \rightarrow \infty} \limsup _{n \rightarrow \infty}\left|\frac{\Sigma_{n}(F, z)}{\Sigma_{n}(1, z)}-\mathbf{E}\left[F\left(e_{s}, 0 \leqslant s \leqslant \Delta\right)\right]\right| \leqslant \frac{4 \varepsilon}{C / 2-2 \varepsilon},
$$

which completes the proof of (3.15) in the nonlattice case.

$\mathrm{S}$ t e p II (The conditioned convergence of $\left(I_{n}(s n) / \sigma \sqrt{n} ; 0 \leqslant s \leqslant \Delta\right.$ ) for $\Delta<1$ in the lattice case). Assume that the law of $\mathscr{L}$ is supported by $\alpha+\beta \mathbf{Z}$ with span $\beta$. Recall that $a_{n}(0)=\alpha n+\beta\lfloor(3 \ln n / 2-\alpha n) / \beta\rfloor$ and that $a_{n}(z)=a_{n}(0)-z$. We use the same notation of Step I. Let us prove

$$
\lim _{\beta \mathbf{Z} \ni z \rightarrow \infty} \limsup _{n \rightarrow \infty}\left|\frac{\Sigma_{n}(F, z)}{\Sigma_{n}(1, z)}-\mathbf{E}\left[F\left(e_{s}, 0 \leqslant s \leqslant \Delta\right)\right]\right|=0 .
$$

Suppose that $z \in \beta \mathbf{Z}$. Whereas the arguments of Step I, we obtain that for any $L \geqslant L_{\varepsilon}, b \geqslant B(L, \eta), z \geqslant K \geqslant K(\eta)$, and $n$ sufficiently large,

$$
\left|\Sigma_{n}(F, z)-\Lambda_{n}^{I I}(F)\right| \leqslant 2 \eta(1+L)^{2}(1+z-K) e^{-z}+\left(e^{K}+\varepsilon(1+z-K)\right) e^{-z},
$$

where

$$
\begin{aligned}
& \Lambda_{n}^{I I}(F)=\Lambda^{I I}(F, z, K, L, b) \\
&:=e^{a_{n}(0)} e^{-z} \mathbf{E}_{\mathbf{Q}}[ F\left(\frac{V\left(w_{\lfloor s n\rfloor}\right)}{\sigma \sqrt{n}} ; 0 \leqslant s \leqslant \Delta\right) \\
& \times f_{L, b}\left(V\left(w_{n-b}-a_{n}(z+L)\right)\right) ; \\
& \min _{0 \leqslant k \leqslant n-b} V\left(w_{k}\right) \geqslant-z+K, \\
&\left.\min _{\Delta n \leqslant k \leqslant n-b} V\left(w_{k}\right) \geqslant a_{n}(z+L)\right] .
\end{aligned}
$$


Under $\mathbf{Q}$, the distribution of $V\left(w_{1}\right)-V\left(w_{0}\right)$ is also supported by $\alpha+\beta \mathbf{Z}$. Let $d=d(L, b):=\beta\lceil(\alpha b-L) / \beta\rceil-\alpha b+L$ and $\lambda_{n}:=n^{3 / 2} e^{-a_{n}(0)}$. Recall that $f_{L, b}$ is well defined in (3.20), it follows from (II) of Lemma 2.2 that

$$
\lim _{n \rightarrow \infty} \lambda_{n} \Lambda_{n}^{I I}(F)=\alpha_{L, b}^{I I} R(z-K) e^{-z} \mathbf{E}\left[F\left(e_{s}, 0 \leqslant s \leqslant \Delta\right)\right] .
$$

where $\alpha_{L, b}^{I I}:=C_{1} \beta \sum_{j \geqslant 0} f_{L, b}(\beta j+d) R_{-}(\beta j+d) \in[0, \infty)$. Observe that $1 \leqslant \lambda_{n} \leqslant e^{\beta}$. Combining with (3.32), we conclude that

$$
\begin{aligned}
& \limsup _{\beta \mathbf{Z} \ni z \rightarrow \infty} \limsup _{n \rightarrow \infty}\left|\frac{e^{z}}{z} \lambda_{n} \Sigma_{n}(F, z)-\alpha_{L, b}^{I I} c_{0} \mathbf{E}\left[F\left(e_{s}, 0 \leqslant s \leqslant \Delta\right)\right]\right| \\
& \leqslant e^{\beta}\left(2 \eta(1+L)^{2}+\varepsilon\right) .
\end{aligned}
$$

We admit for the moment that there exist $0<c_{9}<c_{10}<\infty$ such that $\alpha_{L, b}^{I I} \in\left[c_{9}, c_{10}\right]$ for all $L, b$ large enough. Then take $\varepsilon<c_{9} c_{0} /\left(4 e^{\beta}\right), L=L_{\varepsilon}$, $\eta=\varepsilon /\left(2\left(1+L_{\varepsilon}\right)^{2}\right)$ and $b \geqslant B\left(L_{\varepsilon}, \eta\right)$ so that $e^{\beta}\left(2 \eta(1+L)^{2}+\varepsilon\right)<c_{9} c_{0} / 2 \leqslant$ $\alpha_{L_{\varepsilon}, b}^{I I} c_{0} / 2 \leqslant 2 c_{10} c_{0}$. Note that

$$
\frac{\Sigma_{n}(F, z)}{\Sigma_{n}(1, z)}=\frac{e^{z} \lambda_{n} \Sigma_{n}(F, z) / z}{e^{z} \lambda_{n} \Sigma_{n}(1, z) / z} .
$$

We thus deduce from (3.34) that

$$
\limsup _{\beta \mathbf{Z} \ni z \rightarrow \infty} \limsup _{n \rightarrow \infty}\left|\frac{\Sigma_{n}(F, z)}{\Sigma_{n}(1, z)}-\mathbf{E}\left[F\left(e_{s}, 0 \leqslant s \leqslant \Delta\right)\right]\right| \leqslant \frac{4 \varepsilon}{c_{9} c_{0} / e^{\beta}-2 \varepsilon},
$$

which tends to zero as $\varepsilon \downarrow 0$.

It remains to prove that $\alpha_{L, b}^{I I} \in\left[c_{9}, c_{10}\right]$ for all $L, b$ large enough. Instead of investigating the entire system, we consider the branching random walk killed at 0 . Define

$$
I_{n}^{\text {kill }}:=\inf \left\{V(u):|u|=n, V\left(u_{k}\right) \geqslant 0 \quad \forall 0 \leqslant k \leqslant n\right\},
$$

and we get the following fact from Corollary 3.4 and Lemma 3.6 of [2].

$\mathrm{F}$ a c t 3.2 (see [2]). There exists a constant $c_{11}>0$ such that for any $n \geqslant 1$ and $x, z \geqslant 0$,

$$
\mathbf{P}_{x}\left(I_{n}^{\mathrm{kill}} \leqslant a_{n}(z)\right) \leqslant c_{11}(1+x) e^{-x-z} .
$$

Moreover, there exists $c_{12}>0$ such that for any $n \geqslant 1$ and $z \in\left[0, a_{n}(1)\right]$,

$$
\mathbf{P}\left(I_{n}^{\text {kill }} \leqslant a_{n}(z)\right) \geqslant c_{12} e^{-z} .
$$

Even though Fact 3.2 is proved in [2] under the assumption that the distribution of $\mathscr{L}$ is nonlattice, the lattice case is actually recovered from that proof. 
Analogically, let $m^{k i l l,(n)}$ be the particle chosen uniformly in the set $\left\{u:|u|=n, V(u)=I_{n}^{\text {kill }}, \min _{0 \leqslant k \leqslant n} V\left(u_{k}\right) \geqslant 0\right\}$. Moreover, let $\Sigma_{n}^{\text {kill }}(1, z):=$ $\mathbf{P}\left[I_{n}^{\text {kill }} \leqslant a_{n}(z)\right]$ and $\Pi_{n}^{\text {kill }}(1, z, z, L):=\mathbf{P}\left[I_{n}^{\text {kill }} \leqslant a_{n}(z), m^{\text {kill, }(n)} \in J_{z, z, L}^{\Delta}(n)\right]$. By (3.7) again, we check that for all $L \geqslant L_{\varepsilon}$,

$$
\begin{aligned}
& \left|\Sigma_{n}^{\mathrm{kill}}(1, z)-\Pi_{n}^{\mathrm{kill}}(1, z, z, L)\right| \\
& \leqslant \mathbf{P}\left[\exists|u|=n: V(u) \leqslant a_{n}(z) ; \min _{0 \leqslant k \leqslant n} V\left(u_{k}\right) \geqslant 0 ;\right. \\
& \left.\min _{\Delta n \leqslant k \leqslant n} V\left(u_{k}\right) \leqslant a_{n}(z+L)\right] \\
& \leqslant \varepsilon e^{-z} \text {. }
\end{aligned}
$$

Recounting the arguments of Step (1), one sees that for any $L \geqslant L_{\varepsilon}, b \geqslant$ $B(L, \eta), z \geqslant K(\eta)$, and $n$ sufficiently large,

$$
\left|\Pi_{n}^{\mathrm{kill}}(1, z, z, L)-\Lambda_{n}^{\mathrm{kill}}\right| \leqslant 2 \eta(1+L)^{2} e^{-z},
$$

where

$\Lambda_{n}^{\mathrm{kill}}:=\mathbf{E}_{\mathbf{Q}}\left[f^{\mathrm{kill}}\left(V\left(w_{n-b}\right)\right) ; \min _{0 \leqslant k \leqslant n-b} V\left(w_{k}\right) \geqslant 0, \min _{\Delta n \leqslant k \leqslant n-b} V\left(w_{k}\right) \geqslant a_{n}(z+L)\right]$,

with

$$
\begin{aligned}
f^{\mathrm{kill}}(x):=\mathbf{E}_{\mathbf{Q}_{x}}\left[\frac{e^{V\left(w_{b}\right)} \mathbf{1}_{\left\{V\left(w_{b}\right)=I_{b}^{\mathrm{kill}}\right\}}}{\sum_{|u|=b} \mathbf{1}_{\left\{V(u)=I_{b}^{\mathrm{kill}}, \min _{0 \leqslant j \leqslant b} V\left(u_{j}\right) \geqslant 0\right\}}} ;\right. \\
\left.\min _{0 \leqslant k \leqslant b} V\left(w_{k}\right) \geqslant a_{n}(z+L), V\left(w_{b}\right) \leqslant a_{n}(z)\right] .
\end{aligned}
$$

For $\varepsilon>0$ and $n$ sufficiently large, it has been proved in [2] that

$$
\left|e^{z} \Lambda_{n}^{\mathrm{II}}(1, z, z, L, b)-\Lambda_{n}^{\mathrm{kill}}\right| \leqslant \varepsilon .
$$

Recalling the convergence (3.33) with $K=z$ and $F \equiv 1$, we deduce from (3.39), (3.40), and (3.42) that for any $L \geqslant L_{\varepsilon}, b \geqslant B(L, \eta)$, and $z \geqslant K(\eta)$

$$
\limsup _{n \rightarrow \infty}\left|\lambda_{n} \Sigma_{n}^{\mathrm{kill}}(1, z)-\alpha_{L, b}^{\mathrm{II}} e^{-z}\right| \leqslant e^{\beta}\left(2 \eta(1+L)^{2}+2 \varepsilon\right) e^{-z},
$$

since $R(0)=1$ and $1 \leqslant \lambda_{n} \leqslant e^{\beta}$. Fact 3.2 implies that $c_{12} \leqslant e^{z} \lambda_{n} \mathbf{P}\left(I_{n}^{\mathrm{kill}} \leqslant\right.$ $\left.a_{n}(z)\right) \leqslant c_{11} e^{\beta}$. Hence, we obtain that

$$
c_{12}-e^{\beta}\left(2 \eta(1+L)^{2}+2 \varepsilon\right) \leqslant \alpha_{L, b}^{I I} \leqslant e^{\beta} c_{11}+e^{\beta}\left(2 \eta(1+L)^{2}+2 \varepsilon\right) .
$$

Let $c_{10}:=c_{11} e^{\beta}+c_{12}$ and $c_{9}:=3 c_{12} / 4>0$. For any $\varepsilon<e^{-\beta} c_{12} / 12$, we take $L=L_{\varepsilon}$ and $\eta \leqslant \varepsilon / 2\left(1+L_{\varepsilon}\right)^{2}$. Then $c_{10}>\alpha_{L, b}^{\mathrm{II}} \geqslant c_{9}>0$ for $b \geqslant B\left(L_{\varepsilon}, \eta\right)$. This completes the second step.

$\mathrm{S}$ t e p III (The tightness). Actually, it suffices to prove the following proposition. 
Proposition 3.2. For any $\eta>0$,

$$
\lim _{\delta \rightarrow 0} \limsup _{z \rightarrow \infty} \limsup _{n \rightarrow \infty} \mathbf{P}\left(\sup _{0 \leqslant k \leqslant \delta n}\left|I_{n}(n-k)-I_{n}\right| \geqslant \eta \sigma \sqrt{n} \mid I_{n} \leqslant a_{n}(z)\right)=0 .
$$

The first two steps allow us to obtain the following fact whether the distribution is lattice or nonlattice.

$\mathrm{F}$ a c t 3.3. There exist constants $c_{13}, c_{14} \in(0, \infty)$ such that

$c_{13} \leqslant \liminf _{z \rightarrow \infty} \liminf _{n \rightarrow \infty} \frac{e^{z}}{z} \mathbf{P}\left(I_{n} \leqslant a_{n}(z)\right) \leqslant \limsup _{z \rightarrow \infty} \limsup _{n \rightarrow \infty} \frac{e^{z}}{z} \mathbf{P}\left(I_{n} \leqslant a_{n}(z)\right) \leqslant c_{14}$.

$\mathrm{Pr}$ o of of $\mathrm{Proposition} 3.2$. First, we observe that for any $M \geqslant 1$ and $\delta \in(0,1 / 2)$,

$$
\begin{aligned}
\mathbf{P}\left(\sup _{0 \leqslant k \leqslant \delta n}\left|I_{n}(n-k)-I_{n}\right| \geqslant \delta \sigma \sqrt{n}, I_{n} \leqslant a_{n}(z)\right) \\
\leqslant \mathbf{P}\left(m_{n}^{(n)} \notin J_{z, 0, L}^{1 / 2}(n), I_{n} \leqslant a_{n}(z)\right) \\
\quad+\mathbf{P}\left(I_{n}(n-\lfloor\delta n\rfloor) \geqslant M \sigma \sqrt{\delta n}, I_{n} \leqslant a_{n}(z)\right)+\chi(\delta, z, n) .
\end{aligned}
$$

where $\chi(\delta, z, n):=\mathbf{P}\left(m_{n}^{(n)} \in J_{z, 0, L}^{1 / 2}(n), \quad I_{n}(n-\lfloor\delta n\rfloor) \leqslant M \sigma \sqrt{\delta n}\right.$, $\left.\sup _{0 \leqslant k \leqslant \delta n}\left|I_{n}(n-k)-I_{n}\right| \geqslant \eta \sigma \sqrt{n}\right)$.

It follows from Lemma 3.1 that for any $\varepsilon>0$, if $L \geqslant L_{\varepsilon}, n \geqslant 1$, and $z \geqslant 0$,

$$
\mathbf{P}\left(m_{n}^{(n)} \notin J_{z, 0, L}^{1 / 2}(n), I_{n} \leqslant a_{n}(z)\right) \leqslant(1+\varepsilon(1+z)) e^{-z} .
$$

Then dividing each term of (3.47) by $\mathbf{P}\left(I_{n} \leqslant a_{n}(z)\right)$ yields that

$$
\begin{aligned}
& \mathbf{P}\left(\sup _{0 \leqslant k \leqslant \delta n}\left|I_{n}(n-k)-I_{n}\right| \geqslant \eta \sigma \sqrt{n} \mid I_{n} \leqslant a_{n}(z)\right) \\
& \leqslant \frac{(1+\varepsilon(1+z)) e^{-z}}{\mathbf{P}\left(I_{n} \leqslant a_{n}(z)\right)}+\mathbf{P}\left(I_{n}(n-\lfloor\delta n\rfloor) \geqslant M \sigma \sqrt{\delta n} \mid I_{n} \leqslant a_{n}(z)\right) \\
&+\frac{\chi(\delta, z, n)}{\mathbf{P}\left(I_{n} \leqslant a_{n}(z)\right)} .
\end{aligned}
$$

On the one hand, by Fact 3.3,

$$
\limsup _{z \rightarrow \infty} \limsup _{n \rightarrow \infty} \frac{(1+\varepsilon(1+z)) e^{-z}}{\mathbf{P}\left(I_{n} \leqslant a_{n}(z)\right)} \leqslant \frac{\varepsilon}{c_{13}} .
$$

On the other hand, Steps I and II tell us that for any $1>\delta>0$ and $M \geqslant 1$,

$$
\limsup _{z \rightarrow \infty} \limsup _{n \rightarrow \infty} \mathbf{P}\left[I_{n}(n-\lfloor\delta n\rfloor) \geqslant M \sigma \sqrt{\delta n} \mid I_{n} \leqslant a_{n}(z)\right]=\mathbf{P}\left[e_{1-\delta} \geqslant M \sqrt{\delta}\right],
$$


which, by Chebyshev's inequality, is bounded by

$$
\frac{\mathbf{E}\left[e_{1-\delta}\right]}{M \sqrt{\delta}}=\frac{4 \sqrt{1-\delta}}{M \sqrt{2 \pi}}
$$

Consequently,

$$
\begin{gathered}
\limsup _{z \rightarrow \infty} \limsup _{n \rightarrow \infty} \mathbf{P}\left(\sup _{0 \leqslant k \leqslant \delta n}\left|I_{n}(n-k)-I_{n}\right| \geqslant \eta \sigma \sqrt{n} \mid I_{n} \leqslant a_{n}(z)\right) \\
\leqslant \frac{\varepsilon}{c_{13}}+\frac{2}{M}+\limsup _{z \rightarrow \infty} \limsup _{n \rightarrow \infty} \frac{\chi(\delta, z, n)}{\mathbf{P}\left(I_{n} \leqslant a_{n}(z)\right)} .
\end{gathered}
$$

Let us estimate $\chi(\delta, z, n)$. One sees that

$$
\begin{aligned}
& \chi(\delta, z, n) \\
& \quad \leqslant \mathbf{E}\left[\sum_{|u|=n} \mathbf{1}_{\left\{u \in J_{z, L}^{1 / 2}(n) ; \sup _{0 \leqslant k \leqslant \delta n}\left|V\left(u_{n-k}\right)-V(u)\right| \geqslant \eta \sigma \sqrt{n} ; V\left(u_{n-\lfloor\delta n\rfloor}\right) \leqslant M \sigma \sqrt{\delta n}\right\}}\right] .
\end{aligned}
$$

By Lemma 2.4, it becomes that

$$
\begin{gathered}
\chi(\delta, z, n) \leqslant \mathbf{E}\left[e^{S_{n}} ; S_{n} \leqslant a_{n}(z), \underline{S}_{n} \geqslant-z, \underline{S}_{[n / 2, n]} \geqslant a_{n}(z+L),\right. \\
\left.S_{n-\lfloor\delta n\rfloor} \leqslant M \sigma \sqrt{\delta n}, \sup _{0 \leqslant k \leqslant \delta n}\left|S_{n-k}-S_{n}\right| \geqslant \eta \sigma \sqrt{n}\right] \\
\leqslant n^{3 / 2} e^{-z} \Upsilon(\delta, z, n),
\end{gathered}
$$

where $\Upsilon(\delta, z, n):=\mathbf{P}\left(S_{n} \leqslant a_{n}(z), \underline{S}_{n} \geqslant-z, \underline{S}_{[n / 2, n]} \geqslant a_{n}(z+L), S_{n-\lfloor\delta n\rfloor} \leqslant\right.$ $\left.M \sigma \sqrt{\delta n}, \sup _{0 \leqslant k \leqslant \delta n}\left|S_{n-k}-S_{n}\right| \geqslant \eta \sigma \sqrt{n}, S_{n-\lfloor\delta n\rfloor} \leqslant M \sigma \sqrt{\delta n}\right)$.

Reversing time yields that

$$
\begin{array}{r}
\Upsilon(\delta, z, n) \leqslant \mathbf{P}\left(\underline{S}_{n}^{-} \geqslant-a_{n}(0), \underline{S}_{n / 2}^{-} \geqslant-L,-S_{n} \in\left[-a_{n}(z),-a_{n}(z+L)\right],\right. \\
\left.\sup _{0 \leqslant k \leqslant \delta n}\left|-S_{k}\right| \geqslant \eta \sigma \sqrt{n},-S_{\lfloor\delta n\rfloor} \leqslant M \sigma \sqrt{\delta n}-a_{n}(z+L)\right) .
\end{array}
$$

Applying the Markov property at time $\lfloor\delta n\rfloor$, we obtain that

$$
\Upsilon(\delta, z, n)=\mathbf{E}\left[\Theta\left(-S_{\lfloor\delta n\rfloor}\right) ; \underline{S}_{\delta n}^{-} \geqslant-L, \sup _{0 \leqslant k \leqslant \delta n}\left|-S_{k}\right| \geqslant \eta \sigma \sqrt{n}\right],
$$

where $\Theta(x):=\mathbf{1}_{\left\{x \leqslant M \sigma \sqrt{\delta n}-a_{n}(z+L)\right\}} \mathbf{P}_{x}\left(\underline{S}_{(1 / 2-\delta) n}^{-} \geqslant-L, \underline{S}_{(1-\delta) n}^{-} \geqslant-a_{n}(0)\right.$, $\left.-S_{n-\lfloor\delta n\rfloor} \in\left[-a_{n}(z),-a_{n}(z+L)\right]\right)$. Reversing time again implies that

$$
\begin{gathered}
\Theta(x) \leqslant \mathbf{1}_{\{x \leqslant M \sigma \sqrt{\delta n\}}} \mathbf{P}\left(\underline{S}_{(1-\delta) n} \geqslant-z-L, \underline{S}_{[n / 2,(1-\delta) n]} \geqslant a_{n}(z+2 L),\right. \\
\left.S_{n-\lfloor\delta n\rfloor} \in\left[x+a_{n}(z+L), x+a_{n}(z)\right]\right) .
\end{gathered}
$$


By $(2.10), \Theta(x) \leqslant c_{15}(1+z+L)(1+L)(1+M \sigma \sqrt{\delta n}+2 L) n^{-3 / 2}$. Plugging it into (3.53) and taking $n$ large enough so that $1+2 L<\eta \sigma \sqrt{\delta n}$, we get that

$$
\begin{aligned}
\Upsilon(\delta, z, n) \leqslant & c_{15}(1+z)(1+L)^{2} n^{-3 / 2}(M+\eta) \sigma \sqrt{\delta n} \\
& \times \mathbf{E}\left[\underline{S}_{\delta n}^{-} \geqslant-L, \sup _{0 \leqslant k \leqslant \delta n}\left|-S_{k}\right| \geqslant \eta \sigma \sqrt{n}\right] .
\end{aligned}
$$

Recall that $\chi(\delta, z, n) \leqslant e^{-z} n^{3 / 2} \Upsilon(\delta, z, n)$. We check that

$$
\begin{aligned}
\chi(\delta, z, n) \leqslant & c_{15} e^{-z}(1+z)(1+L)^{2}(M+\eta) \sigma \\
& \times \mathbf{E}_{L}\left[\sup _{0 \leqslant k \leqslant \delta n}\left(-S_{k}\right) \geqslant \eta \sigma \sqrt{n} \mid \underline{S}_{\delta n}^{-} \geqslant 0\right] \\
& \times\left(\sqrt{\delta n} \mathbf{P}_{L}\left[\underline{S}_{\delta n}^{-} \geqslant 0\right]\right) .
\end{aligned}
$$

On the one hand, by Theorem 1.1 of [7], $\mathbf{E}_{L}\left[\sup _{0 \leqslant k \leqslant \delta n}\left(-S_{k}\right) \geqslant \eta \sigma \sqrt{n} \mid \underline{S}_{\delta n}^{-} \geqslant\right.$ $0]$ converges to $\mathbf{P}\left(\sup _{0 \leqslant s \leqslant 1} \mathscr{M}_{s} \geqslant \eta / \sqrt{\delta}\right)$ as $n \rightarrow \infty$. On the other hand, (2.7) shows that $\sqrt{\delta n} \mathbf{P}_{L}\left[\underline{S}_{\delta n}^{-} \geqslant 0\right]$ converges to $C_{-} R_{-}(L)$ as $n \rightarrow \infty$. Therefore,

$$
\begin{aligned}
\limsup _{n \rightarrow \infty} \chi(\delta, z, n) \leqslant & c_{15} e^{-z}(1+z)(1+L)^{2}(M+\eta) \sigma C_{-} R_{-}(L) \\
& \times \mathbf{P}\left(\sup _{0 \leqslant s \leqslant 1} \mathscr{M}_{s} \geqslant \eta / \sqrt{\delta}\right) .
\end{aligned}
$$

Going back to (3.51) and letting $z \rightarrow \infty$, we deduce from Fact 3.3 that

$$
\begin{aligned}
\limsup _{z \rightarrow \infty} \limsup _{n \rightarrow \infty} \mathbf{P}\left(\sup _{0 \leqslant k \leqslant \delta n}\left|I_{n}(n-k)-I_{n}\right| \geqslant \eta \sigma \sqrt{n} \mid I_{n} \leqslant a_{n}(z)\right) \\
\leqslant \frac{\varepsilon}{c_{13}}+\frac{2}{M} \\
\quad+\frac{c_{15}(1+L)^{2}(M+\eta) \sigma C_{-} R_{-}(L) \times \mathbf{P}\left(\sup _{0 \leqslant s \leqslant 1} \mathscr{M}_{s} \geqslant \eta / \sqrt{\delta}\right)}{c_{13}} .
\end{aligned}
$$

Notice that $\mathbf{P}\left(\sup _{0 \leqslant s \leqslant 1} \mathscr{M}_{s} \geqslant \eta / \sqrt{\delta}\right)$ decreases to 0 as $\delta \downarrow 0$. Take $M \geqslant 2 / \varepsilon$. We conclude that for any $0<\varepsilon<c_{13}$,

$$
\begin{gathered}
\limsup _{\delta \rightarrow 0} \limsup _{z \rightarrow \infty} \limsup _{n \rightarrow \infty} \mathbf{P}\left(\sup _{0 \leqslant k \leqslant \delta n}\left|I_{n}(n-k)-I_{n}\right| \geqslant \eta \sigma \sqrt{n} \mid I_{n} \leqslant a_{n}(z)\right) \\
\leqslant \frac{\varepsilon}{c_{13}}+\varepsilon
\end{gathered}
$$

which completes the proof of Proposition 3.2. And Proposition 3.1 is thus proved. 
4. Proof of Theorem 1.1. Let us prove the main theorem now. It suffices to prove that for any continuous functional $F: D([0,1], \mathbf{R}) \rightarrow[0,1]$, we have

$$
\lim _{n \rightarrow \infty}\left|\mathbf{E}\left[F\left(\frac{I_{n}(\lfloor s n\rfloor)}{\sigma \sqrt{n}} ; 0 \leqslant s \leqslant 1\right)\right]-\mathbf{E}\left[F\left(e_{s}, 0 \leqslant s \leqslant 1\right)\right]\right|=0 .
$$

P r o o f of (4.1). Define for $A \geqslant 0$,

$$
\mathscr{Z}[A]:=\left\{u \in \mathbb{T}: V(u) \geqslant A>\max _{k<|u|} V\left(u_{k}\right)\right\}
$$

For any particle $u \in \mathscr{Z}[A]$, there is a subtree rooted at $u$. If $|u| \leqslant n$, let

$$
I_{n}(u):=\min _{v \geqslant u,|v|=n} V(v) .
$$

Moreover, assume $m_{n}^{u}$ is the particle uniformly chosen in the set $\{|v|=n: v \geqslant$ $\left.u, V(v)=I_{n}(u)\right\}$. Similarly, we write $\llbracket, m_{n}^{u} \rrbracket:=\left\{\varnothing=: m_{0}^{u}, m_{1}^{u}, \ldots, m_{n}^{u}\right\}$. The trajectory leading to $m_{n}^{u}$ is denoted by $\left\{V\left(m_{k}^{u}\right) ; 0 \leqslant k \leqslant n\right\}$. Let $\omega_{A}$ be the particle uniformly chosen in $\left\{u \in \mathscr{Z}[A]:|u| \leqslant n, I_{n}(u)=I_{n}\right\}$.

Let $\mathscr{Y}_{A}:=\left\{\max _{u \in \mathscr{Z}[A]}|u| \leqslant M, \max _{u \in \mathscr{Z}[A]} V(u) \leqslant M\right\}$. Then for any $\varepsilon>0$, there exist $M:=M(A, \varepsilon)$ large enough such that $\mathbf{P}\left(\mathscr{Y}_{A}^{c}\right) \leqslant \varepsilon$. It follows that

$$
\begin{aligned}
& \mid \mathbf{E}\left[F\left(\frac{I_{n}(\lfloor s n\rfloor)}{\sigma \sqrt{n}} ; 0 \leqslant s \leqslant 1\right)\right]-\mathbf{E}\left[F\left(\frac{I_{n}(\lfloor s n\rfloor)}{\sigma \sqrt{n}} ; 0 \leqslant s \leqslant 1\right) ;\right. \\
& \left.\quad \mathscr{Y}_{A},\left|I_{n}-a_{n}(0)\right| \leqslant A / 2\right] \mid \\
& \leqslant \varepsilon+\mathbf{P}\left[\left|I_{n}-a_{n}(0)\right| \geqslant \frac{A}{2}\right] .
\end{aligned}
$$

We then check that for $n \geqslant M$,

$$
\begin{aligned}
\mathbf{E} & {\left[F\left(\frac{I_{n}(\lfloor s n\rfloor)}{\sigma \sqrt{n}} ; 0 \leqslant s \leqslant 1\right) ; \mathscr{Y}_{A},\left|I_{n}-a_{n}(0)\right| \leqslant \frac{A}{2}\right] } \\
& =\mathbf{E}\left[\sum_{u \in \mathscr{Z}[A]} \mathbf{1}_{\left(u=\omega_{A}\right)} F\left(\frac{V\left(m_{\lfloor s n\rfloor}^{u}\right)}{\sigma \sqrt{n}} ; 0 \leqslant s \leqslant 1\right) ; \mathscr{Y}_{A},\left|I_{n}-a_{n}(0)\right| \leqslant \frac{A}{2}\right] .
\end{aligned}
$$

Define another trajectory $\left\{\widetilde{V}\left(m_{k}^{u}\right) ; 0 \leqslant k \leqslant n\right\}$ as follows:

$$
\widetilde{V}\left(m_{k}^{u}\right):= \begin{cases}V(u) & \text { if } k<|u| \\ V\left(m_{k}^{u}\right) & \text { if }|u| \leqslant k \leqslant n .\end{cases}
$$


It follows that

$$
\begin{aligned}
\mathbf{E}\left[F\left(\frac{I_{n}(\lfloor s n\rfloor)}{\sigma \sqrt{n}} ; 0 \leqslant s \leqslant 1\right) ; \mathscr{Y}_{A},\left|I_{n}-a_{n}(0)\right| \leqslant \frac{A}{2}\right] \\
=\mathbf{E}\left[\sum_{u \in \mathscr{Z}[A]} \mathbf{1}_{\left(u=\omega_{A}\right)} F\left(\frac{\tilde{V}\left(m_{\lfloor s n\rfloor}^{u}\right)}{\sigma \sqrt{n}} ; 0 \leqslant s \leqslant 1\right) ; \mathscr{Y}_{A},\left|I_{n}-a_{n}(0)\right| \leqslant \frac{A}{2}\right] \\
\quad+o_{n}(1),
\end{aligned}
$$

where $o_{n}(1) \rightarrow 0$ as $n$ goes to infinity.

Define the sigma-field $\mathscr{G}_{A}:=\sigma\left\{\left(u, V(u), I_{n}(u)\right) ; u \in \mathscr{Z}[A]\right\}$. Note that on $\mathscr{Y}_{A}, I_{n}=\min _{u \in \mathscr{Z}[A]} I_{n}(u)$ as long as $n \geqslant M$. One sees that $\mathscr{Y}_{A} \cap\left\{\mid I_{n}-\right.$ $\left.a_{n}(0) \mid \leqslant A / 2\right\}$ is $\mathscr{G}_{A}$-measurable for all $n$ large enough. Thus,

$$
\begin{gathered}
\mathbf{E}\left[\sum_{u \in \mathscr{Z}[A]} \mathbf{1}_{\left(u=\omega_{A}\right)} F\left(\frac{\tilde{V}\left(m_{\lfloor s n\rfloor}^{u}\right)}{\sigma \sqrt{n}} ; 0 \leqslant s \leqslant 1\right) ; \mathscr{Y}_{A},\left|I_{n}-a_{n}(0)\right| \leqslant \frac{A}{2}\right] \\
=\mathbf{E}\left[\sum_{u \in \mathscr{Z}[A]} \mathbf{1}_{\left(u=\omega_{A}\right)} \mathbf{E}\left[F\left(\frac{\tilde{V}\left(m_{\lfloor s n\rfloor}^{u}\right)}{\sigma \sqrt{n}} ; 0 \leqslant s \leqslant 1\right) \mid \mathscr{G}_{A}, u=\omega_{A}\right]\right. \\
\left.\mathscr{Y}_{A},\left|I_{n}-a_{n}(0)\right| \leqslant A / 2\right] .
\end{gathered}
$$

Further, we notice by the branching property that conditioned on $\{(u, V(u)) ; u \in \mathscr{Z}[A]\}$, the subtrees generated by $u \in \mathscr{Z}[A]$ are independent copies of the original one, started from $V(u)$, respectively. Therefore, given $\mathscr{Y}_{A} \cap\left\{\left|I_{n}-a_{n}(0)\right| \leqslant A / 2\right\}$,

$$
\begin{aligned}
& \mathbf{1}_{\left(u=\omega_{A}\right)} \mathbf{E}\left[F\left(\frac{\tilde{V}\left(m_{\lfloor s n\rfloor}^{u}\right)}{\sigma \sqrt{n}} ; 0 \leqslant s \leqslant 1\right) \mid \mathscr{G}_{A}, u=\omega_{A}\right] \\
& \quad=\mathbf{1}_{\left(u=\omega_{A}\right)} \mathbf{E}\left[F\left(\frac{I(\lfloor s(n-|u|)\rfloor)}{\sigma \sqrt{n-|u|}} ; 0 \leqslant s \leqslant 1\right) \mid I_{n-|u|} \leqslant a_{n}\left(-r_{u}\right)\right]+o_{n}(1),
\end{aligned}
$$

where $r_{u}:=\min \left\{\min _{v \in \mathscr{Z}[A] \backslash\{u\}} I_{n}(v)-a_{n}(0), A / 2\right\}-V(u)$ is independent of $I_{n-|u|}$. Thus, (4.6) becomes

$$
\begin{gathered}
\mathbf{E}\left[F\left(\frac{I_{n}(\lfloor s n\rfloor)}{\sigma \sqrt{n}} ; 0 \leqslant s \leqslant 1\right) ; \mathscr{Y}_{A},\left|I_{n}-a_{n}(0)\right| \leqslant \frac{A}{2}\right] \\
=\mathbf{E}\left[\sum_{u \in \mathscr{Z}[A]} \mathbf{1}_{\left(u=\omega_{A}\right)} \mathbf{E}\left[F\left(\frac{I(\lfloor s(n-|u|)\rfloor)}{\sigma \sqrt{n-|u|}} ; 0 \leqslant s \leqslant 1\right) \mid I_{n-|u|} \leqslant a_{n}\left(-r_{u}\right)\right] ;\right. \\
\left.\mathscr{Y}_{A},\left|I_{n}-a_{n}(0)\right| \leqslant \frac{A}{2}\right]+o_{n}(1) .
\end{gathered}
$$

The event $\mathscr{Y}_{A} \cap\left\{\left|I_{n}-a_{n}(0)\right| \leqslant A / 2\right\}$ ensures that $A / 2+M \geqslant-r_{u} \geqslant A / 2$. The conditioned convergence has been given in Proposition 3.1. We need a slightly stronger version here. 
According to Proposition 3.1, for any $\varepsilon>0$, there exists $z_{\varepsilon}>0$ such that for all $z \geqslant z_{\varepsilon}$,

$$
\begin{aligned}
& \limsup _{n \rightarrow \infty} \mid \mathbf{E}\left[F\left(\frac{I_{n}(\lfloor s n\rfloor)}{\sigma \sqrt{n}} ; 0 \leqslant s \leqslant 1\right) \mid I_{n} \leqslant a_{n}(z)\right] \\
&-\mathbf{E}\left[F\left(e_{s}, 0 \leqslant s \leqslant 1\right)\right] \mid<\varepsilon .
\end{aligned}
$$

Thus, for any $z \geqslant z_{\varepsilon}$, there exists $N_{z} \geqslant 1$ such that for any $n \geqslant N_{z}$,

$$
\begin{aligned}
& \mid \mathbf{E}\left[F\left(\frac{I_{n}(\lfloor s n\rfloor)}{\sigma \sqrt{n}} ; 0 \leqslant s \leqslant 1\right) \mid I_{n} \leqslant a_{n}(z)\right] \\
& -\mathbf{E}\left[F\left(e_{s}, 0 \leqslant s \leqslant 1\right)\right] \mid<2 \varepsilon .
\end{aligned}
$$

Take $A=2 z_{\varepsilon}$ and $K=M$. We say that for $n$ sufficiently large,

$$
\begin{gathered}
\sup _{z \in\left[z_{\varepsilon}, z_{\varepsilon}+K\right]} \mid \mathbf{E}\left[F\left(\frac{I(\lfloor s(n)\rfloor)}{\sigma \sqrt{n}} ; 0 \leqslant s \leqslant 1\right) \mid I_{n} \leqslant a_{n}(z)\right] \\
-\mathbf{E}\left[F\left(e_{s}, 0 \leqslant s \leqslant 1\right)\right] \mid \leqslant 3 \varepsilon .
\end{gathered}
$$

In the lattice case, (4.11) follows immediately. We only need to prove it in the nonlattice case.

Recall that

$$
\Sigma_{n}(F, z)=\mathbf{E}\left[F\left(\frac{I_{n}(\lfloor s n\rfloor)}{\sigma \sqrt{n}} ; 0 \leqslant s \leqslant 1\right) ; I_{n} \leqslant a_{n}(z)\right]
$$

with $0 \leqslant F \leqslant 1$. Then, for any $\ell>0$ and $z \geqslant 0$,

$$
\begin{gathered}
\left|\frac{\Sigma_{n}(F, z)}{\Sigma_{n}(1, z)}-\frac{\Sigma_{n}(F, z+\ell)}{\Sigma_{n}(1, z+\ell)}\right| \\
\leqslant\left|\frac{\Sigma_{n}(F, z)-\Sigma_{n}(F, z+\ell)}{\Sigma_{n}(1, z)}\right|+\left|\frac{\Sigma_{n}(F, z+\ell)}{\Sigma_{n}(1, z)}-\frac{\Sigma_{n}(F, z+\ell)}{\Sigma_{n}(1, z+\ell)}\right| \\
=\frac{1}{\Sigma_{n}(1, z)}\left(\mid \begin{array}{c}
\left|\Sigma_{n}(F, z)-\Sigma_{n}(F, z+\ell)\right| \\
\left.\quad+\frac{\Sigma_{n}(F, z+\ell)}{\Sigma_{n}(1, z+\ell)}\left|\Sigma_{n}(1, z+\ell)-\Sigma_{n}(1, z)\right|\right) .
\end{array}\right.
\end{gathered}
$$

Since $0 \leqslant F \leqslant 1$, the two inequalities

$$
\begin{aligned}
& \left|\Sigma_{n}(F, z)-\Sigma_{n}(F, z+\ell)\right| \\
& \quad=\mathbf{E}\left[F\left(\frac{I_{n}(\lfloor s n\rfloor)}{\sigma \sqrt{n}} ; 0 \leqslant s \leqslant 1\right) ; a_{n}(z+\ell)<I_{n} \leqslant a_{n}(z)\right] \\
& \quad \leqslant \mathbf{P}\left(a_{n}(z+\ell)<I_{n} \leqslant a_{n}(z)\right),
\end{aligned}
$$


and

$$
\frac{\Sigma_{n}(F, z+\ell)}{\Sigma_{n}(1, z+\ell)} \leqslant 1
$$

hold. Note also that $\left|\Sigma_{n}(1, z+\ell)-\Sigma_{n}(1, z)\right|=\mathbf{P}\left(a_{n}(z+\ell)<I_{n} \leqslant a_{n}(z)\right)$. It follows that

$$
\begin{aligned}
\left|\frac{\Sigma_{n}(F, z)}{\Sigma_{n}(1, z)}-\frac{\Sigma_{n}(F, z+\ell)}{\Sigma_{n}(1, z+\ell)}\right| & \leqslant 2 \frac{\mathbf{P}\left(a_{n}(z+\ell)<I_{n} \leqslant a_{n}(z)\right)}{\mathbf{P}\left(I_{n} \leqslant a_{n}(z)\right)} \\
& =2-2 \frac{\mathbf{P}\left(I_{n} \leqslant a_{n}(z+\ell)\right)}{\mathbf{P}\left(I_{n} \leqslant a_{n}(z)\right)} .
\end{aligned}
$$

In view of Fact 3.1 , we take $3 \ln n / 2-\Lambda_{\varepsilon^{\prime}} \geqslant \ell+z>z \geqslant \Lambda_{\varepsilon^{\prime}}$ so that for any $n \geqslant N_{\varepsilon^{\prime}}$,

$$
\frac{\mathbf{P}\left(I_{n} \leqslant a_{n}(z+\ell)\right)}{\mathbf{P}\left(I_{n} \leqslant a_{n}(z)\right)} \geqslant \frac{\left(C-\varepsilon^{\prime}\right)(z+\ell) e^{-z-\ell}}{\left(C+\varepsilon^{\prime}\right) z e^{-z}} \geqslant \frac{C-\varepsilon^{\prime}}{C+\varepsilon^{\prime}} e^{-\ell} .
$$

For $\varepsilon^{\prime}=C \varepsilon / 8>0$, we choose $\zeta=\varepsilon / 4$ so that $\left(C-\varepsilon^{\prime}\right) e^{-\zeta} /\left(C+\varepsilon^{\prime}\right) \geqslant$ $1-\varepsilon / 2$. As a consequence, for any $\Lambda_{\varepsilon^{\prime}} \leqslant z \leqslant 3 \ln n / 2-\Lambda_{\varepsilon^{\prime}}-\zeta, 0 \leqslant \ell \leqslant \zeta$, and $n \geqslant N_{\varepsilon^{\prime}}$,

$$
\left|\frac{\Sigma_{n}(F, z)}{\Sigma_{n}(1, z)}-\frac{\Sigma_{n}(F, z+\ell)}{\Sigma_{n}(1, z+\ell)}\right| \leqslant 2\left(1-\frac{C-\varepsilon^{\prime}}{C+\varepsilon^{\prime}} e^{-\ell}\right) \leqslant \varepsilon .
$$

For $\varepsilon>0, z_{\varepsilon}$ can be chosen so that $\left[z_{\varepsilon}, z_{\varepsilon}+K\right] \subset\left[\Lambda_{\varepsilon^{\prime}}, 3 \ln n / 2-\Lambda_{\varepsilon^{\prime}}\right]$ for $n \geqslant e^{K} N_{\varepsilon^{\prime}}$. For any integer $0 \leqslant j \leqslant\lceil K / \zeta\rceil$, let $z_{j}:=z_{\varepsilon}+j \zeta$. Then $\left[z_{\varepsilon}, z_{\varepsilon}+K\right] \subset \cup_{0 \leqslant j \leqslant\lceil K / \zeta\rceil}\left[z_{j}, z_{j+1}\right]$. Take $N_{\varepsilon}^{\prime}=\max _{0 \leqslant j \leqslant\lceil K / \zeta\rceil}\left\{N_{z_{j}}, e^{K} N_{\varepsilon^{\prime}}\right\}$. By (4.10) and (4.15), we conclude that for any $n \geqslant N_{\varepsilon}^{\prime}$,

$$
\begin{gathered}
\sup _{z \in\left[z_{\varepsilon}, z_{\varepsilon}+K\right]} \mid \mathbf{E}\left[F\left(\frac{I_{n}(\lfloor s n\rfloor)}{\sigma \sqrt{n}} ; 0 \leqslant s \leqslant 1\right) \mid I_{n} \leqslant a_{n}(z)\right] \\
-\mathbf{E}\left[F\left(e_{s}, 0 \leqslant s \leqslant 1\right)\right] \mid \\
\leqslant \sup _{0 \leqslant j \leqslant\lceil K / \zeta\rceil}\left|\frac{\Sigma_{n}\left(F, z_{j}\right)}{\Sigma_{n}\left(1, z_{j}\right)}-\mathbf{E}\left[F\left(e_{s}, 0 \leqslant s \leqslant 1\right)\right]\right| \\
+\sup _{0 \leqslant j<\lceil K / \zeta\rceil} \sup _{z_{j} \leqslant z \leqslant z_{j+1}}\left|\frac{\Sigma_{n}(F, z)}{\Sigma_{n}(1, z)}-\frac{\Sigma_{n}\left(F, z_{j}\right)}{\Sigma_{n}\left(1, z_{j}\right)}\right| \leqslant 3 \varepsilon .
\end{gathered}
$$

We continue to prove the main theorem. Since $\sum_{u \in \mathscr{Z}[A]} \mathbf{1}_{\left(u=\omega_{A}\right)}=1$, we deduce from (4.8) and (4.11) that for $n$ sufficiently large,

$$
\begin{aligned}
& \mid \mathbf{E}\left[F\left(\frac{I_{n}(\lfloor s n\rfloor)}{\sigma \sqrt{n}} ; 0 \leqslant s \leqslant 1\right) ; \mathscr{Y}_{A},\left|I_{n}-a_{n}(0)\right| \leqslant \frac{A}{2}\right] \\
& \quad-\mathbf{E}\left[F\left(e_{s}, 0 \leqslant s \leqslant 1\right)\right] \mid
\end{aligned}
$$




$$
\begin{aligned}
& \leqslant 3 \varepsilon \mathbf{P}\left(\mathscr{Y}_{A} ;\left|I_{n}-a_{n}(0)\right| \leqslant \frac{A}{2}\right)+o_{n}(1)+\mathbf{P}\left(\mathscr{Y}_{A}^{c}\right)+\mathbf{P}\left(\left|I_{n}-a_{n}(0)\right| \geqslant \frac{A}{2}\right) \\
& \leqslant 4 \varepsilon+o_{n}(1)+\mathbf{P}\left(\left|I_{n}-a_{n}(0)\right| \geqslant \frac{A}{2}\right) .
\end{aligned}
$$

Going back to (4.3), we conclude that for $n$ large enough,

$$
\begin{gathered}
\left|\mathbf{E}\left[F\left(\frac{I_{n}(\lfloor s n\rfloor)}{\sigma \sqrt{n}} ; 0 \leqslant s \leqslant 1\right)\right]-\mathbf{E}\left[F\left(e_{s}, 0 \leqslant s \leqslant 1\right)\right]\right| \\
\leqslant 5 \varepsilon+2 \mathbf{P}\left(\left|I_{n}-a_{n}(0)\right| \geqslant \frac{A}{2}\right)+o_{n}(1) .
\end{gathered}
$$

Let $n$ go to infinity and then make $\varepsilon \downarrow 0$. Therefore,

$$
\begin{aligned}
\limsup _{n \rightarrow \infty}\left|\mathbf{E}\left[F\left(\frac{I_{n}(\lfloor s n\rfloor)}{\sigma \sqrt{n}} ; 0 \leqslant s \leqslant 1\right)\right]-\mathbf{E}\left[F\left(e_{s}, 0 \leqslant s \leqslant 1\right)\right]\right| \\
\leqslant \limsup _{z \rightarrow \infty} \limsup _{n \rightarrow \infty} 2 \mathbf{P}\left(\left|I_{n}-a_{n}(0)\right| \geqslant z\right) .
\end{aligned}
$$

It remains to show that $\limsup _{z \rightarrow \infty} \lim \sup _{n \rightarrow \infty} \mathbf{P}\left(\left|I_{n}-a_{n}(0)\right| \geqslant z\right)=0$. Because of Fact 3.3, it suffices to prove that

$$
\limsup _{z \rightarrow \infty} \limsup _{n \rightarrow \infty} \mathbf{P}\left(I_{n} \geqslant a_{n}(0)+z\right)=0 .
$$

In the nonlattice case, Theorem 1.1 of [2] implies it directly. In the lattice case, we see that for $n$ large enough,

$$
\mathbf{P}\left(I_{n} \geqslant a_{n}(0)+z\right) \leqslant \mathbf{E}\left[\prod_{u \in \mathscr{Z}[A]}\left(1-\Phi_{u}(z, n)\right) ; \mathscr{Y}_{A}\right]+\varepsilon,
$$

with $\Phi_{u}(z, n):=\mathbf{P}\left(I_{n-|u|} \leqslant a_{n}(V(u)-z)\right)$. Take $A=2 z$ here. Then it follows from Fact 3.3 that for $n$ large enough and for any particle $u \in \mathscr{Z}[A]$,

$$
\Phi_{u}(z, n) \geqslant \frac{c_{13}}{2}(V(u)-z) e^{z-V(u)} \geqslant \frac{c_{13}}{4} V(u) e^{z-V(u)} .
$$

(4.18) hence becomes

$$
\begin{aligned}
\limsup _{n \rightarrow \infty} \mathbf{P}\left(I_{n} \geqslant a_{n}(0)+z\right) & \leqslant \mathbf{E}\left[\prod_{u \in \mathscr{Z}[A]}\left(1-\frac{c_{13}}{4} V(u) e^{z-V(u)}\right) ; \mathscr{Y}_{A}\right]+\varepsilon \\
& \leqslant \mathbf{E}\left[\exp \left(-\frac{c_{13}}{4} e^{z} \sum_{u \in \mathscr{Z}[A]} V(u) e^{-V(u)}\right)\right]+\varepsilon .
\end{aligned}
$$

It has been proved that as $A$ goes to infinity, $\sum_{u \in \mathscr{Z}[A]} V(u) e^{-V(u)}$ converges almost surely to some limit $D_{\infty}$, which is strictly positive on the set of nonextinction of $\mathbb{T}$, (see (5.2) in [2]). We end up with

$$
\limsup _{z \rightarrow \infty} \limsup _{n \rightarrow \infty} \mathbf{P}\left(I_{n} \geqslant a_{n}(0)+z\right) \leqslant \varepsilon,
$$

which completes the proof of Theorem 1.1. 
Acknowledgments. I am grateful to my Ph. D. advisor Zhan Shi for his advice and encouragement.

\section{REFERENCES}

1. Addario-Berry L., Reed B. Minima in branching random walks. - Ann. Probab., 2009, v. 37, № 3, p. 1044-1079.

2. Aïdékon E. Weak convergence of the minimum of a branching random walk. ArXiv:1101.1810, 2011.

3. Aïdékon E., Berestycki J., Brunet É., Shi Z. The branching Brownian motion seen from its tip. Arxiv:1104.3738v2, 2011.

4. Aïdékon E., Shi Z. The Seneta-Heyde scaling for the branching random walk, preprint, 2010.

5. Biggins J. D., Kyprianou A. E. Fixed points of the smoothing transform: the boundary case. - Electron. J. Probab., 2005, v. 10, № 17, p. 609-631.

6. Caravenna $F$. A local limit theorem for random walks conditioned to stay positive. Probab. Theory Related Fields, 2005, v. 133, № 4, p. 508-530.

7. Caravenna $F$., Chaumont $L$. Invariance principles for random walks conditioned to stay positive. - Ann. Inst. Henri Poincaré Probab. Statist., 2008, v. 44, № 1, p. 170190.

8. Chauvin B., Rouault A. KPP equation and supercritical branching Brownian motion in the subcritical speed area. Application to spatial trees. - Probab. Theory Related Fields, 1988, v. 80, № 2, p. 299-314.

9. Durrett R., Iglehart D., Miller D. Weak convergence to Brownian meander and Brownian excursion. - Ann. Probab., 1977, v. 5, № 1, p. 117-129.

10. Феллер В. Введение в теорию вероятностей и ее применения. Т. 2, 2-е изд. М.: Мир, 1984, 752 с.

11. $H u Y$., Shi Z . Minimal position and critical martingale convergence in branching random walks, and directed polymers on disordered trees. - Ann. Probab., 2009, v. 37 , № 2 , p. $742-789$.

12. Imhof J.-P. Density factorizations for Brownian motion, meander and the threedimensional Bessel process, and applications. - J. Appl. Probab., 1984, v. 21, p. 500510 .

13. Козлов М.В. Об асимптотике вероятности невырождения критических ветвящихся процессов в случайной среде. - Теория вероятн. и ее примен., 1976 т. 21 , в. 4 , с. $813-825$.

14. Kyprianou A. Travelling wave solutions to the K-P-P equation: alternatives to Simon Harris' probabilistic analysis. - Ann. Inst. H. Poincaré Probab. Statist., 2004, v. 40, № 1 , p. 53-72.

15. Lyons $R$. A simple path to Biggins' martingale convergence for branching random walk. In: Classical and Modern Branching Processes. Eds. Athreya K. B., Jagers P. New York: Springer, 1997, v. 84, p. 217-221.

16. Revuz D., Yor M. Continuous Martingales and Brownian Motion. 3rd ed. New York: Springer-Verlag, 2005, $602 \mathrm{p}$. 